\title{
Homosexuellenunterdrückung in der bürgerlichen Gesellschaft
}

\section{Thorsten Graf, Mimi Steglitz (1)}

Die Haltung der Kommunisten und Sozialisten in Deutschland zur Frage der Homosexuellenunterdrückung war bisher fast ausnahmslos von Vorurteilen und Unwissenheit gekennzeichnet. Die Solidarität mit den Opfern der Homosexuellenunterdrückung, den Opfern des berüchtigten $\S 175$ StGB und der Ächtung durch das „gesunde Volksempfinden“" blieb -- wenn überhaupt von Solidarität die Rede sein konnte - fast immer eine halbe Sache. Vorwissenschaftliche Entscheidungen darüber, was das Natürliche, Gesunde, Normale usw. sei, behindern heute wie früher eine nüchterne Beurteilung dieser Frage, die eine zahlenmäßig nicht unbeträchtliche Gruppe von Frauen und Männern betrifft. Wendet man die Prozentzahlen der Kinsey-Berichte auf die BRD an, dann beträgt z. B. schon allein die Zahl der ausschließlich homosexuell Ausgerichteten ca. zwei Millionen. Darüber hinaus haben nach Kinsey $37 \%$ der Männer und $13 \%$ der Frauen in der weißen US-Bevölkerung physische homosexuelle Erfahrungen (2).

An die Stelle der nahezu totalen Tabuisierung trat nicht in gleichem Maße wie im Bürgertum bei den Sozialisten die Verdammung der Homosexualität als Laster, Entartung, Perversität usw. Stattdessen wurde schon recht früh die modernere Form des antihomosexuellen Vorurteils vertreten, wonach homosexuelles Verhalten als Krankheit gewertet wird und Homosexuelle demnach bemitleidenswerte Kranke sind, die man allenfalls trotz ihrer ,,unglücklichen Veranlagung“ akzeptiert. Diese Inkonsequenz und Halbheit in der Überwindung des Vorurteils, die auch je nach den Umständen Rückfall in die Tabuisierung oder gar in antihomosexuellen Terror einschließt, ist eines der Themen dieses Aufsatzes.

Zunächst soll anhand einiger Beispiele der in der Geschichte der deutschen Arbeiterbewegung anzutreffende Standpunkt zur Homosexuellenunterdrückung charakterisiert werden. Darüber hinaus wird, wegen ihrer vorbildartigen Bedeutung für die deutsche Arbeiterbewegung die Entwicklung in der Sowjetunion herangezogen. Daran anschließende Überlegungen zur Funktion der Homosexuellenunterdrückung in der bürgerlichen Gesellschaft, sowie einige Bemerkungen zum Klassencharakter der frühen Emanzipationsbewegung der Homosexuellen in Deutschland sollen die Perspektiven der seit einigen Jahren wieder auflebenden Homosexuellenbewegung in der BRD und in Westberlin klären helfen.

Es geht also um den Versuch einer angemessenen Beurteilung dieser Frage, die von der gegenwärtig unter Sozialisten immer noch weitgehend geübten Tabuisierung des Problems genauso weit entfernt ist wie von einer Überschätzung seiner Bedeu-

1 Wir veröffentlichen diesen Aufsatz unter Pseudonymen, nicht, weil wir ein eventuelles Berufsverbot für Schwule fürchten, sondern weil wir uns der entscheidenderen Bedrohung durch das Berufsverbot für ,Radikale“ im öffentlichen Dienst aussetzen würden.

A. Kinsey u. a.: Das sexuelle Verhalten der Frau. Berlin, Frankfurt a. M. 1954, S. 367 
tung. Das Tabu, mit dem diese Frage durch die „normale“, sexuell mehr oder weniger angepaßte Mehrheit der Sozialisten bisher bedacht wurde, hatte in der Überbewertung durch die Homosexuellen selbst eine ähnlich fragwürdige Ergänzung gefunden. Diese schlechte Wechselwirkung zu stören, ist die Absicht der schwulen Verfasser des vorliegenden Aufsatzes.

Die wichtige Frage nach dem Zusammenhang zwischen den vielfältigen Formen und Funktionen der allgemeinen Sexualunterdrückung in der bürgerlichen Gesellschaft und dem speziellen Fall der Homosexuellenunterdrückung wird im Folgenden nicht erörtert. Nur auf der Basis einer materialistischen Sozialpsychologie, die allenfalls bruchstuickhaft in Sicht ist, könnte zu dieser Frage, die auf die Bedeutung der Geschlechterrollen für den bürgerlichen Herrschaftszusammenhang abzielt, mehr gesagt werden als einerseits Friedrich Engels bereits angedeutet hat oder andererseits von bürgerlichen Ideologen mit anscheinend unerschöpflichem Einfallsreichtum herumspekuliert wird.

Deshalb konzentrieren sich die folgenden Ausführungen auf Bereiche, aus denen sich schon jetzt ohne Riickgriff auf die komplexe sozialpsychologische Frages stellung praktisch-politische Schlußfolgerungen und Einschätzungen herleiten lassen. Sicher kann das bisherige Verhältnis zwischen der Homosexuellenemanzipationsbewegung und der Arbeiterbewegung nur vollständig erklärt und verändert werden bei Berïcksichtigung sozialpsychologischer Probleme. Eine solche Vollständigkeit wird hier nicht beansprucht. Der Anspruch ist vielmehr, einen in der marxistischen Diskussion seit mehreren Jahrzehnten nahezu vollständig tabuisierten Bereich überhaupt erst beim Namen zu nennen und dazu beizutragen, daß ein Anfang gemacht wird.

Zur Terminologie im vorliegenden Text ist zu bemerken, daß der Ausdruck Homosexualität immer nur zur Bezeichnung gleichgeschlechtlichen Sexualverhaltens verwendet wird. Homosexuellenunterdrückung wird dort manifest, wo das homosexuelle Verhalten eines Menschen bekannt wird. Ob er lebenslang und ausschließlich homosexuell ist, oder ob er sich daneben auch heterosexuell verhalten kann, ist unter dem Gesichtspunkt der gesellschaftlichen Unterdrückung hauptsächlich von quantitativer Bedeutung: Je weniger Möglichkeiten einem zur Verfügung stehen, sich als „Normaler“ zu tarnen, desto unerbittlicher ist die Diskriminierung. Sie trifft in unterschiedlichem Ausmaß jeden, der sich homosexuell verhält, wobei es prinzipiell auch nebensächlich ist, ob es sich um einen Mann oder um eine Frau handelt.

Selbst der neuerdings Verbreitung findende Versuch, seine homosexuellen Neigungen dadurch anzudeuten, daß man sich als ,bisexuell“ bezeichnet, schützt ebenso unzureichend vor Diskriminierung wie die Versicherung, man sei gar nicht homosexuell, sondern „homophil“. Wichtiger als Geschlechtszugehörigkeit, Häufigkeit und Ausschließlichkeit dürfte für Grad und Form der Unterdrückung sowieso die Klassenlage des sich homosexuell Verhaltenden sein. 


\section{Arbeiterbewegung und Homosexuellenunterdrïckung}

Am 16. Oktober 1929 wurde im Strafrechtsausschuß des deutschen Reichstags beschlossen, daß „Unzucht zwischen Männern“ im neuen Strafrecht nicht mehr vorkommen sollte. Die dem $\S 175$ entsprechende Bestimmung wurde aufgrund des gemeinsamen Vorgehens der kommunistischen und sozialdemokratischen Abgeordneten ersatzlos gestrichen. Nur der Sieg des Faschismus 1933 verhinderte, daß diese Entscheidung rechtsgültig wurde.

Wenn man sich im Protokoll der der Abstimmung vorausgegangenen Debatte den Redebeitrag des KP-Abgeordneten Maslowski ansieht, dann läßt sich daran die Widersprüchlichkeit und politische Halbheit in der Haltung der Linken exemplarisch veranschaulichen:

„Abgeordneter Maslowski [ . . wendete] sich gegen die moralischen Qualifikationen und Begriffe, die der Abgeordnete Schetter wieder erwähnt habe. Gewiß sei Homosexualität eine Triebabartung. Wolle man sie aber bestrafen, dann müsse man auch alle anderen Abartungen, wie Onanie, bestrafen [...]. Sei das Argument des Reichsjustizministers richtig, daß durch ärztliche Behandlung eine Heilung der Homosexualität möglich sei, dann solle man nicht nach dem Strafrichter, sondern nach dem Arzt rufen" (3).

Auf der einen Seite sieht Maslowski sehr rich tig, daß homosexuelles Verhalten nicht schädlicher oder nützlicher ist als beispielsweise Onanie, andererseits akzeptiert er aber im Argument des Reichsjustizministers den Begriff „Heilung" in Bezug auf Homosexualität. Homosexualität ist demnach für ihn bestenfalls eine Krankheit, denn nur in Bezug auf eine Krankheit ist es sinnvoll, von Heilung zu sprechen.

Die Auffassung, daß Homosexuelle deshalb nicht strafrechtlich verfolgt werden dürfen, weil Homosexualität eine Krankheit ist, hat durchaus eine Tradition in der Arbeiterbewegung. Dies läßt sich weiterhin beispielhaft zeigen an der einzigen größeren Reichstagsdebatte über den $\S 175$ am 31. 3. 1905. Als der SPD-Abgeordnete Thiele die kühne Außerung wagte: „Ich für meine Per'son mag nicht einmal zugeben, daß das etwas Krankhaftes ist, sondern es ist eben nur eine Abweichung der Natur von den üblichen Mustern, die sie hervorbringt" (4), wurde von seinem Kollegen von Vollmar wenig später erklärt, „daß Kollege Thiele wie jeder andere Kollege ohne Rücksicht auf die Parteiangehörigkeit, der in dieser Angelegenheit spricht, in dieser Sache lediglich persönlich Stellung nimmt, und die Sozialdemokratie so wenig wie irgendeine andere Partei mit dieser Sache irgend etwas zu schaffen hat. (Sehr richtig! links. Hört! Hört! rechts)“ (5). Thiele und von Vollmar gehörten bekanntlich zum rechten Flügel der SPD. Von den Linken wurde zu dieser Frage durchweg völliges Stillschweigen bewahrt. Ein Sonderfall ist lediglich August Bebel.

3 Reichstag. IV. Wahlperiode 1928, 21. Ausschuß (Reichsstrafgesetzbuch), 85. Sitzung am 16. 10. 1929, S. 8

4 Reichtstagsprotokolle, Bd. 204, S. 5829

5 a. a. O., S. 5839 
Als erster Redner überhaupt trat er schon im Jahre 1898 im Reichtstag für die Abschaffung des $\S 175$ ein. Er unterzeichnete als einer der ersten die Petition für die Abschaffung des $\S 175$, die von der ersten deutschen Homosexuellenorganisation, dem Wissenschaftlich-humanitären Komitee, an den Reichtstag gerichtet wurde (6). Aber auch bei Bebel findet sich wieder jene Halbheit gegenüber den eigenen Vorurteilen. Sind für ihn homosexuelle Handlungen keine Krankheit, so sind es doch „Widernatïrlichkeiten“ und ,erschreckende Tatsachen“. In seinem Buch ,Die Frau und der Sozialismus" heißt es unter ,5. Verbrechen gegen die Sittlichkeit und Geschlechtskrankheiten":

„Auch abgesehen von jenen, welchen die Liebe zum eigenen Geschlecht (die Homosexualität) angeboren ist, verfallen viele in die Widernatürlichkeiten des griechischen Zeitalters. Die Männerliebe ist viel weiter verbreitet, als sich die meisten von uns träumen lassen; darüber könnten die geheimen Akten mancher Polizeibüros erschreckende Tatsachen veröffentlichen. Aber auch unter den Frạuen leben die Widernatürlichkeiten des alten Griechenland in stärkerem Maße wieder auf" (7).

Es ist insgesamt äußerst schwierig, Stellungnahmen aus der Arbeiterbewegung zur Homosexualität ausfindig zu machen. Das Tabu, mit dem die sexuelle Frage belegt war, traf und trifft in verstärktem Maß die Homosexualität. Wenn das Thema berührt wurde, dann sollten vermutlich die negativen und abwertenden Formulierungen unter anderem auch Selbstschutzfunktionen erfüllen: der Verdacht, man sei selbst „so", wird am besten abgewehrt, wenn man sich rhetorischer Muster wie Mitleid-mit-bedauernswerten-Kranken oder Ehrliche-Entrüstung-über-Lasterhaftigkeit bedient. In besonders krasser Form war dies anläßlich des Krupp-Skandals 1902 der Fall, der durch einen Artikel des Zentralorgans ,Vorwärts“ ausgelöst wurde. Der Großindustrielle F. A. Krupp wurde in dem Artikel „Krupp auf Capri“ als ein aufgrund seiner Homosexualität besonders scheußliches Exemplar der herrschenden Klasse angeprangert. Es heißt dort u. a.:

„Das grauenhafte Bild kapitalistischer Beeinflussung wird dadurch nicht sonderlich milder, daß man weiß, es handelt sich um einen pervers veranlagten Mann. Denn das Mitleid, das das Opfer eines verhängnisvollen Natur-Irrtums verdient, muß versagen, wenn die Krankheit zu ihrer Befriedigung Millionen in ihre Dienste stellt" (8).

Bei diesen Überlegungen muß jedoch immer berücksichtigt werden, daß die Arbeiterparteien, die SPD wie die KPD, sich von allen politischen Kräften am konsequentesten - trotz aller Vorbehalte - für die Beseitigung von Sondergesetzen gegen homosexuelle Männer und für die Verhinderung solcher Gesetze gegen homosexueller Frauen engagierten und daß ferner die konzentrierten Kräfte des antihomosexuellen Verfolgungswahns und der archaischen Ängste vor dem Aussterben des deutschen Volkes wegen sich seuchenartig ausbreitender Homosexualität vor allem

6 Vgl. Jahrbuch für sexuelle Zwischenstufen, Jg. 1, 1899, S. 272 ff. Dort ist die vollständige Reichs̀tagsrede Bebels vom 13.1.1898 wiedergegeben.

7 Zit. nach der 162. Aufl., Berlin (DDR) 1973, S. 238

8 "Vorwärts" vom 15. 11. 1902. Zit. nach: Jahrbuch für sexuelle Zwischenstufen, Jg. 5, 1903. Dort findet sich eine ausführliche Darstellung des Verlaufs des Krupp-Skandals. 
in der herrschenden Klasse konzentriert waren. Die relative Vorurteilslosigkeit in der SPD hängt auch damit zusammen, daß diese Partei letztlich der fortgeschrittenste politische Repräsentant der Bourgeoisie war und ist.

Folgende Äußerung des kommunistischen Rechtsanwalts Felix Halle in seinem Buch „Geschlechtsleben und Strafrecht“, Berlin 1931, weisen trotz ihrer Vereinfachung und Idealisierung auf eine reale Tendenz hin:

„Das klassenbewußte Proletariat, nicht beeinflußt durch die Ideologie des Besitzes und befreit von der Ideologie der Kirchen, geht in der Frage des Geschlechtslebens und auch an das Problem der Homosexualität mit jener Vorurteilslosigkeit heran, die ihm die Erkenntnis der allgemeinen sozialen Zusammenhänge ermöglicht [...] Es betrachtet diese Vorgänge gemäß den naturwissenschaîtlichen Erkenntnissen der neueren Zeit als Sonderform geschlechtlicher Befriedigung und verlangt gegenüber diesen Formen des Geschlechtslebens die gleiche Freiheit und Begrenzung wie im Verkehr der verschiedenen Geschlechter untereinander, d. h. Schutz für die Geschlechtsunreifen gegen Angriffe, Schutz der Bestimmungen über den eigenen Körper und schließlich die Achtung vor den Rechten Außenstehender" (S. 72 f.).

Lenin hat sich wie Marx und Engels (9) nie zur Frage der Homosexuellenunterdrückung geäußert.

Daß die Sowjetunion in ihrer Funktion als Vorbild für die deutschen Kommunisten wenigstens bis zum Beginn der terroristischen Homosexuellenverfolgungen im Jahre 1934 auch eine sehr ambivalente Haltung zur Homosexuellenfrage einnahm, soll an dem dafür repräsentativen Artikel „Homosexualität“ von M. Serejskij in der Großen Sowjetenzyklopädie aus dem Jahre 1929 belegt werden. „Heilung“ und „Prophylaxe“ sind auch dort die nächstliegenden Assoziationen gewesen:

„... Die Prognose für Homosexualität ist relativ schlecht. Eine Heilung (izlečenie) gelingt nur in einem unbedeutenden Teil der Fälle und das hauptsächlich da, wo bisexuelle Tendenzen vorliegen. Die wichtigste Aufgabe ist die Prophylaxe: eine korrekte Sexualerziehung, Koedukation in der Schule. In Fällen, wo ein bedeutender Anteil äußerer Faktoren vorliegt, hat die Psychotherapie einen gewissen Erfolg, insbesondere Psychoanalyse, Hypnose, Sport."

Daß auch heute bei Sozialisten solche Fehleinschätzungen und Halbheiten in der Úberwindung antihomosexueller Vorurteile anzutreffen sind, läßt sich leicht belegen. Allerdings ist heute fast noch mehr als früher unter Sozialisten die Tabuisierung des Themas die Regel, Stellungnahmen sind die Ausnahme. Nur zwei Beispiele seien hier angeführt. R. Reiche schrieb in seinem unter fortschrittlichen Studenten sehr verbreiteten Buch „Sexualität und Klassenkampf" (Frankfurt a. M. 1968):

„Kulturell stellt die Homosexualität eine Form des Steckenbleibens auf dem Weg von der biologisch ursprünglich richtungslosen Bisexualität zur gesellschaftlich geforderten genitalen Heterosexualität dar ... Individuell müssen mit Homosexualität stets bestimmte Schädigungen der Triebstruktur zusammenfallen." (S. 116)

9 Im „Ursprung der Familie“" berichtet Engels lediglich von den Männern im antiken Griechenland, daß ,sie versanken in die Widerwärtigkeit der Knabenliebe und ihre Götter entwürdigten wie sich selbst durch den Mythus von Ganymed" (MEW 21, S. 67). Hier muß gewissermaßen als mildernder Umstand berücksichtigt werden, daß eine Sexualwissenschaft erst nach Engels' Tod sich zu entwickeln begann. 
Später wird sich noch zeigen, daß Reiche hier als Adept W. Reichs und H. Marcuses argumentiert.

In der Tageszeitung „Die Wahrheit“ der Sozialistischen Einheitspartei Westberlin erschien am 23. 1. 1974 ein Artikel „Neues Sexualstrafrecht - die alte Unmoral bleibt", in dem es zur neuesten Modifizierung des $\S 175$ StGB heißt:

„Das neue Sexualstrafrecht enthält freilich auch einige Regelungen, die zu begrußßen sind und längst überfällig waren. So wurde die Strafbarkeit der Homosexualität zwischen Männern über 18 Jahren beseitigt, und nicht länger kriminalisiert, was in den Bereich der Medizin gehört."

Wie schon 45 Jahre früher der oben zitierte Genosse Maslowski, der „Heilung“ statt Strafe wünschte, erhofft die SEW von der „Medizin“ die Beseitigung des so unerfreulichen Homosexuellenproblems.

$\mathrm{Da}$ die Überzeugung, homosexuelles Verhalten sei ein Krankheitssymptom und Homosexuelle bedürften wegen dieses "Leidens" besonderer Toleranz und Nachsicht, wissenschaftlich nicht fundiert ist (10) und auf ähnlichen vorwissenschaftlichen, von tradierten Ideologien beeinflußten Entscheidungen beruht wie der $\S 175$, erscheint es zunächst als sinnvoll, einige Funktionen und Entwicklungstendenzen dieser Anschauung in der bürgerlichen Gesellschaft zu untersuchen. Hat die gesellschaftliche und rechtliche Ächtung homosexuellen Verhaltens eine notwendige Funktion für die bürgerliche Gesellschaft? Oder haben wir es hier mit einem Relikt vorkapitalistischer Traditionen zu tun, das nur so lange in der kapitalistischen Gesellschaft mitgeschleppt wird, weil es kein ernsthaftes Hindernis für die Kapitalverwertung bildet? Enthält die Homosexuellenunterdrückung dem Kapital nützliche Momente? Wie im einzelnen hat man sich den Zusammenhang zwischen bürgerlicher Gesellschaft und Homosexuellenunterdrückung vorzustellen?

\section{Homosexuellenunterdrückung und bürgerliche Gesellschaft}

Trotz aller neueren Lockerungen der Sexualmoral im Kapitalismus steht homosexuelles Verhalten in einem mehr oder weniger ausgeprägten Widerspruch zu den herrschenden Normen. Dieser Widerspruch kann so extreme Formen annehmen wie im Hitlerfaschismus, wo der industriemäßige Massenmord an Homosexuellen betrieben wurde (11). Die bisher abgeschwächteste Form der Homosexuellenunterdrückung finden wir dagegen in einigen Großstädten kapitalistischer Länder (New York, Amsterdam usw., auch Westberlin), in denen den Homosexuellen eine gettoartige Freizeitsubkultur aus Bars, Dampfbädern und anderen

10 Vgl. Rudolf Klimmer: Die Homosexualität als biologisch-soziologische Zeitfrage. 3. Aufl., Hamburg 1965, bes. S. 67 ff. und 271 f.; und Evelyn Hooker: The adjustment of the male overt homosexual. In; Journal of Projective Techniques, Jg. 21 (1957), S. $18-31$

11 Vgl. hierzu u. a. Wolfgang Harthauser, Der Massenmord an Homosexuellen im Dritten Reich, in: Willhart Schlegel (Hrsg.), Das große Tabu, München 1967, und Rudolf Heß, Kommandant in Auschwitz, München 1963. In beiden Texten wird der KZ-Terror gegen Homosexuelle dokumentiert. 
Treffpunkten, der mehr oder weniger ungehinderte Pornographiekonsum usw. gewährt wird (12). Eine volle Gleichberechtigung der Homosexuellen im bürgerlichdemokratischen Sinne hat es bisher in keinem kapitalistischen Land gegeben; wenn keine staatlichen Sondergesetze gegen Homosexuelle existierten, so war die Unterdrückung und gesellschaftliche Benachteiligung durch andere Mechanismen gewährleistet.

So sind z. B. in der letzten Zeit bekannt gewordene Versuche in Westberlin und in der BRD, Homosexuelle mit einem Berufsverbot zu belegen, etwa weil sie im Erziehungsbereich angeblich ein ,zu großes Risiko“ darstellen, Unterdrückungsmaßnahmen gegen am Arbeitsplatz offen als Homosexuelle Auftretende (13). Diese Berufsverbote werden ähnlich wie die Diskriminierung homosexueller Frauen durchgeführt, ohne daß entsprechende Strafgesetze bestehen (14). Es ist jedoch zu fragen, ob daraus wirklich der Schluß gezogen werden kann, daß Homosexuellenunterdrückung ein Wesensmerkmal der bürgerlichen Gesellschaft im Unterschied zu früheren und späteren Gesellschaftsformationen ist. Eine solche Schlußfolgerung findet sich z. B. bei R. Reiche und M. Dannecker, wenn sie schreiben:

„Das Verhalten der Homosexuellen ist objek tiv eine Zersetzung der bürgerlichen Sexualmoral" (15).

Auch in der Grundsatzerklärung der Homosexuellen Aktion Westberlin (HAW) wird die Einschränkung des Sexualverhaltens in bezug auf Homosexualität als „bürgerlich“ angesehen: „Die praktizierte Homosexualität stellt bereits eine Durchbrechung der bürgerlichen Einschränkung des Sexualverhaltens ( . . ) dar" (16).

Zunächst fällt es demgegenüber nicht schwer, Beispiele aus anderen Gesellschaftsformationen anzuführen, wo Homosexualität entweder in allen Formen, in einigen Formen oder bei bestimmten Gruppen die gleiche Bewertung erfährt wie Heterosexualität. So gibt es z. B. „primitive“ Gesellschaften, die strengste Homosexuellenunterdrückung kennen, wie die Trobrianden in Nordost-Neuguinea (17)

12 Vgl. z. B. Martin Hoffmann, Die Welt der Homosexuellen, Frankfurt a. M. 1971; Magnus Hirschfeld, Berlins drittes Geschlecht, Berlin und Leipzig 1905; Zur neuesten Entwicklung in USA: H. Tilton, Die Herren in den Bädern, in: Frankfurter Rundschau vom 2. 2.1974

13 Erst im Herbst 1974 erregte der Fall des homosexuellen Hauptschullehrers R. Koepp in Westberlin einiges Aufsehen, der von einem CDU-Stadtrat entlassen wurde. In erster Instanz wurde vom Arbeitsgericht die Entlassung vorläufig rückgängig gemacht, allerdings nicht mit der ausdrücklichen Begründung, daß Homosexualität im Schuldienst kein Entlassungsgrund sein darf.

14 Das derzeit geltende antihomosexuelle Strafgesetz in der DDR ( $\$ 151$ des StGB der DDR) ist hier eine der wenigen Ausnahmen; es sieht Strafen für Frauen und Männer vor.

15 M. Dannecker, R. Reiche, Aufstiegsbewußt, freizeitbewußt, normal, Ms. einer Rundfunksendung im SFB am 18. 11. 1971, S. 1

16 „Vorläufige Grundsatzerklärung“c der HAW vom 7.11. 1971, S. 1. Wir nehmen an, daß sowohl Dannecker/Reiche wie auch die HAW den Begriff , bürgerlich" im Sinne des wissenschaftlichen Sozialismus verwenden und nicht in seiner alltagssprachlichen Bedeutung.

17 Vg1. B. Malinowski, Geschlecht und Verdrängung in primitiven Gesellschaften, 2. Aufl., Reinbek 1970, S. 93 
und andererseits solche, wo Homosexuelle sozial geachtet waren (18). „Nach einer Übersicht von Ford und Beach über 76 Stammeskulturen wird in 27 dieser Gemeinwesen homosexuelles Verhalten bestraft oder zumindest mißbilligt, während es in 49 Gemeinwesen akzeptiert wird"(19). Es gab Feudalgesellschaften, wo Homosexualität in gleicher Weise anerkannt war wie Heterosexualität. Das war in China und Japan der Fall (20). Es gab andere, wo sie bekämpft wurde, wie z. B. in Europa:

„Ein verschärfter Kampf gegen die Homosexualität wurde vor allem unter dem Einfluß des Christentums eröffnet. Konstantin sah Homosexualität in einem Gesetz von $326 \mathrm{n}$. Chr. als ein Verbrechen an, daß mit den ausgesuchtesten Strafen belegt werden müsse. Valentian setzte sich für den Feuertod als Strafe ein. Justitian drohte auch die Todesstrafe durch das Schwert an und hob besonders hervor, daß sich Homosexuelle in der Gewalt des Teufels befänden und daß sie verantwortlich seien für schreckliche Plagen [... Das erste deutsche Reichsstrafgesetzbuch: die Constitutio Criminalis Carolina von 1532, Kaiser Karls peinliche Gerichtsordnung [sah vor, daßs] Sodomie, männliche und weibliche Homosexualität mit dem Feuertod bestraft werden. Erst allmählich tritt [ ... ] an die Stelle des Feuertods die Enthauptung" (21).

Eine einfache Einordnung der Homosexuellenunterdrückung als bürgerliche Erscheinung scheint uns schon aufgrund dieser Beispiele zu undifferenziert zu sein. Im Folgenden soll der Nachweis versucht werden, daß die zusätzliche Unterdrückung der Homosexuellen im Zuge einer Verschärfung und Nivellierung des allgemeinen Drucks im Kapitalismus notwendig im Rückgang begriffen ist; daß die bürgerliche Gesellschaft selbst objektive Bedingungen für die Integration der homosexuellen Minderheit in einem von besonderen Widersprüchen gekennzeichneten Prozeß hervorbringt; es soll nachgewiesen werden, daß die Integration der Homosexuellen nicht im Widerspruch zu kapitalistischen Produktionsverhältnissen steht; weiterhin soll auf einige Tatsachen hingewiesen werden, die die Tendenz zur Enttabuisierung der Homosexualität und zur rationaleren Auseinandersetzung mit diesen Formen abweichenden Verhaltens belegen; schließlich soll belegt werden, daß die homosexuelle Emanzipationsbewegung - als relativ selbständige Bewegung selbst ein Produkt der bürgerlichen Gesellschaft - mit ihren politischen Zielen nicht notwendigerweise den bürgerlichen Rahmen durchbricht.

Daraus wären dann für Sozialisten und Homosexuelle einige Konsequenzen abzuleiten:

- die aktive Solidarität der Sozialisten mit der homosexuellen Minderheit im Kampf gegen ihre Sonderunterdrückung würde auch einschließen, daß die homosexuellen Sozialisten in den verschiedenen Organisationen ermutigt wer-

18 M. Mead, Mann und Weib, Stuttgart 1955, S. 145: „In vielen amerikanischen Indianerstämmen war der Mann, der sich wie eine Frau kleidete und lebte, eine anerkannte soziale Institution."

19 K. Freund, Homosexualität, Reinbek 1969, S. 60; s. a. C. S. Ford, F. A. Beach, Formen der Sexualität, Reinbek 1969, S. 138 f.

20 S. Jwaya, Nan sho k' (Die Päderastie in Japan), in: Jahrbuch für sexuelle Zwischenstufen, Jg. 4, 1902, S. 265-272

21 J. Baumann, Paragraph 175, Berlin und Neuwied 1968, S. 25 ff. Dort auch weitere Hinweise und genaue Quellenangaben. 
den, sich nicht länger selbst zu verleugnen und sich in gleicher Weise zu ihren abweichenden Sexualinteressen zu bekennen, wie es für die „,normalen“ Genossen selbstverständlich ist.

- bei den Homosexuellen die Einsicht, daß ihr Kampf für Akzeptierung und gegen die Sonderunterdrückung notwendig borniert bleiben mußs, wenn sie nicht darüber hinaus ihrem im wesentlichen durch ihre mehrheitliche Lage als ausgebeutete Lohnarbeiter bedingtes Interesse am Sozialismus praktisch-politischen Ausdruck verleihen. Der vorläufige Charakter dieser Ausführungen, vor allem wegen des Ausblendens der sozialpsychologischen Aspekte, sollte im folgenden jedoch bedacht werden.

Die bürgerliche Gesellschaft unterscheidet sich bekanntlich von früheren Klassengesellschaften dadurch, daß die Ausbeutung in der Form der Lohnarbeit stattfindet und durch diese Form zugleich mystifiziert wird. Das Verhältnis zwischen Kapitalisten und Lohnarbeitern ist kein direktes Herrschafts- und Knechtschaftsverhältnis mehr wie z. B. das zwischen Sklavenhaltern und Sklaven, sondern die Ausbeutung findet statt unter dem Schein von Freiheit und Gleichheit der Geld- und Warenbesitzer. Die Arbeitskraft der Lohnarbeiter erscheint als Ware wie jede andere.

Die Entstehung des Kapitalismus ist kein einmaliger Akt, sondern ein langwieriger widersprüchlicher Prozeß, in dem sich die Bourgeoisie vom Feudalismus emanzipiert, ihren eigenen Staat, ihre eigenen Gesetze und ihre eigene Moral durchsetzt. Je mehr sich die bürgerliche Gesellschaft entwickelt, je mehr also die gesellschaftliche Arbeit sich verallgemeinert, die kapitalistische Warenproduktion sich ausweitet, desto mehr wird die ,,Fortvegetation altertümlicher, überlebter Produktionsweisen mit ihrem Gefolge von zeitwiderigen gesellschaftlichen und politischen Verhältnissen" (22) eingeschränkt und schließlich ganz beseitigt.

Die Darstellung der Anatomie der bürgerlichen Gesellschaft durch Marx im „Kapital" unterstellt jene voll ausgebildete, ihrem Begriff entsprechende bürgerliche Gesellschaft. Marx:

„In der Theorie wird vorausgesetzt, daß die Gesetze der kapitalistischen Produktionsweise sich rein entwickeln. In der Wirklichkeit besteht immer nur Annäherung; aber diese Annähẹrung ist umso größer, je mehr ihre Verunreinigung und Verquickung mit Resten früherer ökonomischer Zustände beseitigt ist“" (23).

Die voll entwickelte kapitalistische Produktionsweise ist nun gekennzeichnet durch die volle Verwirklichung von formaler Gleichheit und Freiheit der Lohnarbeiter als Voraussetzung fuir ihre Unfreiheit und Ausbeutung im Arbeitsprozeß. Diese Freiheit und Gleichheit äußert sich u. a. in der für den Kapitalismus günstigsten Staatsform, der parlamentarischen Demokratie, im allgemeinen Wahlrecht, in der Freizügigkeit und auch im Abbau von Benachteiligungen und Privilegien, die aus vorkapitalistischer Zeit überkommen waren. Je mehr sich der Kapitalismus durchsetzt, desto stärker werden alle al tertümlichen Ideologien und Traditionen zurückgedrängt. Un-

MEW 23, S. 15

23 MEW 25, S. 184 
ter kapitalistischen Bedingungen ist dies jedoch auch immer daran gekoppelt, daß „die Masse des Elends, des Drucks, der Knechtschaft, der Entartung, der Ausbeutung", d. h. die psychische und in Krisenzeiten auch die physische Verelendung der Arbeiterklasse wächst; die Vereinzelung und Isolation, die Konkurrenz aller mit allen nimmt immer menschenfeindlichere Formen an, wenn nicht jene andere Tendenz sich in gleichem Maße entwickelt, die Marx an der selben Stelle im „Kapital“" benennt: die wachsende „Empörung der stets anschwellenden und durch den Mechanismus des kapitalistischen Produktionsprozesses selbst geschulten, vereinten und organisierten Arbeiterklasse" (24).

Je weniger dieser Prozeß allerdings fortgeschritten ist, desto leichter wird es der Bourgeoisie, gewisse altertümliche und noch immer lebendige Anschauungen in Zeiten der Krise zu aktivieren, die sich zur Ablenkung vom Klassenkampf einsetzen lassen. So konnte z. B. der Antisemitismus in Deu tschland deshalb eine so wichtige ideologische Stützfunktion in den 30er Jahren übernehmen, weil dèr deutsche Kapitalismus sich relativ spät entwickelt hatte und eine so christlich-feudalistische Ideologie wie der Antisemitismus noch im Denken und Fühlen der Menschen - besonders im noch starken Kleinbürgertum - lebendig war. Von grundlegender Bedeutung ist natürlich nicht die Reaktivierung absterbender Ideologien, wenn eine Krise der Kapitalherrschaft und die Machteroberung durch das Proletariat abgewehrt werden soll. Entscheidend ist vielmehr die Abschaffung der bürgerlich-demokratischen Herrschaftsform zugunsten offen terroristischer Formen, die Zerschlagung der Organisationen der Arbeiterklasse und die Liquidierung ihrer Führer. Deshalb hatte auch im Hitlerfaschismus die Reaktivierung der im allmählichen Rückgang begriffenen antihomosexuellen Ressentiments und die Einleitung einer Homosexuellenverfolgung im großen Maßstab allenfalls eine unterstützende Funktion. Speziell bei den Nazis war die Haltung gegenüber den Homosexuellen bis 1935, dem Jahr der Verschärfung des $\S 175$, nicht völlig eindeutig, Zwar war eine der ersten Taten der Faschisten im Jahre 1933 die Zerstörung des Hirschfeldschen Instituts für Sexualforschung in Berlin als Symbol für den Emanzipationskampf der Homosexuellen; und schon 1928 hieß es in der Antwort der NSDAP auf die Frage einer Homosexuellen-Zeitschrift:

„Alles, was unser Volk entmannt, zum Spielball seiner Feinde macht, lehnen wir ab ... Wir verwerfen daher jede Unzucht, vor allem die mannmännliche Liebe, weil sie uns der letzten Möglichkeiten beraubt, jemals unser Volk von den Sklavenketten zu befreien, unter denen es jetzt frohnt" (25).

Daß jedoch eine um Anpassung bemühte und stramme Männlichkeit zur Schau stellende Homosexualität, wie sie z. B. von dem SA-Führer Röhm verkörpert wurde, mindestens am Anfang durchaus akzeptierbar war, läßt sich an einem 1934 in Berlin

MEW 23, S. 790 f.

25 Zit. bei R. Klare, Homosexualität und Strafrecht, Hamburg 1937, S. 149 im Dokumentenanhang. Auf die gleiche Anfrage antwortete die KPD: ,[...] Es erübrigt sich wohl, noch extra zu betonen, daß wir auch in Zukunft für die Beseitigung dieser Paragraphen (175 und 218) den schärfsten Kampf führen werden." (a. a. O., S. 148) 
erschienenen Buch des Faschisten Kurt Hildebrand „Norm Entartung Verfall“"verdeutlichen. Hildebrand war ein Mitglied der Gruppe um den mit dem Faschismus sympathisierenden homosexuellen Schriftsteller Stefan George. Er feiert die ,erotische“ Knabenliebe, die schon in Sparta und Athen zur „Steigerung der Männlichkeit" dazugehört habe , und fährt dann fort:

„Wje man diese Formen mit jener Homosexualität, von der man heute so viel Wesens macht, verwechseln kann, ist unbegreiflich. Diese stammt umgekehrt aus weibischen Männerkreisen, sie wirkt dem Männlichen (Kriegerischen und Geistigen) entgegen, sie hat sexuelle, keine erotischen Tendenzen. Dies moderne Unwesen ist der Entartung verdächtig und des Verfalls gewiß.“ (S. 207)

Des Verfalls, der Ausrottung in den KZs waren schließllich nahezu alle Homosexuellen gewiß, ob ,weibisch“ oder nicht.

\section{Exkurs: Der vereinzel te Einzelne, die Kleinfamilie und die Homosexualität}

Auf den ersten Blick scheint homosexuelles Verhalten nicht nur im Gegensatz zur Heterosexualität zu stehen. Von der Homosexualität scheint sogar geradezu eine Gefahr für heterosexuelle Verhaltensweisen und damit für die Erzeugung von Nachwuchs und für die Aufzucht in den traditionellen Formen auszugehen. Homosexuellenunterdrückung scheint nach dieser oberflächlichen Sichtweise unabdingbar für die Gewährleistung einer heterosexuell-monogamen Lebensform, die die Vererbung des Privateigentums bzw. die beständige Neuerzeugung ausbeutbarer Arbeitskräfte sichert. Tatsächlich wurde nicht selten in dieser Richtung von Befürwortern der Homosexuellenunterdrückung argumentiert.

Solchen Vorstellungen liegt die Annahme zugrunde, daß von der Homosexualität eine Verführungs- oder Ansteckungsgefahr ausgeht, die den davon Betroffenen jedes heterosexuelle Verhalten verleidet und sie zu ausschließlich homosexuellem Verhalten zwingt. Allein schon die Kinseyschen Forschungsergebnisse, nach denen die große Mehrzahl der Befragten mit homosexuellen Erfahrungen sich auch weiterhin heterosexuell betätigt, können die Abwegigkeit jener Annahme verdeutlichen. Im folgenden sollen darüberhinausgehend einige Überlegungen angestellt werden, ob nicht andere, vom Kapitalismus selbst hervorgebrachte Sachverhalte die Verwirklichung traditioneller Formen heterosexueller Geschlechtsliebe zunehmend erschweren.

Die vorhin angeführte Marxsche Aussage über die Entwicklungstendenz des Kapitalismus, eine wachsende „Masse des Elends, des Drucks, der Knechtschaft, der Entartung der Ausbeutung" zu erzeugen, soll jetzt in einem hierfür wichtigen Punkt präzisiert werden, wobei wir zur Verdeutlichung unseres Gedankengangs zunächst von der anderen Seite dieser Entwicklung abstrahieren, daß „der Fortschritt der Industrie ... an die Stelle der Isolierung der Arbeiter durch die Konkurrenz ihre revolutionäre Vereinigung durch die Assoziation (setzt) ... die Expropriation weni- 
ger Usurpatoren durch die Volksmassen“ (26):

Der ,vereinzelte Einzelne“ oder die ,atomistische Privatperson ist ein charakteristisches Produkt der kapitalistischen Gesellschaft. In dem Maße wie der Kapitalismus sich entwickelt, beruhen die Verhältnisse der Menschen untereinander immer weniger auf der „Unreife des individuellen Menschen, der sich von der Nabelschnur des natürlichen Gattungszusammenhangs mit anderen noch nicht losgerissen hat" (27). Auch unmittelbare Herrschafts- und Knechtschaftsverhältnisse werden mehr und mehr von den kapitalistischen Ausbeutungsbeziehungen verdrängt:

„Im Fortgang der kapitalistischen Gesellschaft entwickelt sich eine Arbeiterklasse, die aus Erziehung, Tradition, Gewohnheit die Anforderungen jener Produktionsweise als selbstverständliche Naturgesetze anerkennt ... Außerökonomische unmittelbare Gewalt wird zwar immer noch angewandt, aber nur ausnahmsweise" (28).

Für die im Kapitalismus typische Prägung von Beziehungen, die die Menschen miteinander aufnehmen, ist die Zirkulation wesentlich:

,Die Individuen treten sich nur als Eigentümer von Tauschwerten gegenüber, als solche, die sich ein gegenständliches Dasein füreinander durch ihr Produkt, die Ware, gegeben haben. Ohne diese objektive Vermittlung haben sie keine Beziehung zueinander, vom Standpunkt des in der Zirkulation vor sich gehenden sozialen Stoffwechsels aus betrachtet. Sie existieren nur sachlich füreinander" (29).

Dieser Standpunkt des in der Zirkulation vor sich gehenden sozialen Stoffwechsels ist allerdings kein zum Zweck der Analyse willkürlich gewählter, es ist der wirkliche Standpunkt der Waren- und Geldbesitzer im Kapitalismus. Weiterhin ist die Sphäre der Zirkulation kein dem kapitalistischen Produktionsprozeß äußerlicher Bereich, sondern verleiht ihm die für den Kapitalismus wesentliche Form und ist zudem der Ursprungsort für die zur Funktion des kapitalistischen Produktionsprozesses notwendigen verkehrten Bewußtseinsformen. So ist z. B. die aus der Zirkulation entspringende Verwandlung von Wert und Preis der Arbeitskraft in die Form des Arbeitslohns von entscheidener Wichtigkeit:

,Auf dieser Erscheinungsform (der Lohnform), die das wirkliche Verhältnis unsichtbar macht und gerade sein Gegenteil zeigt, beruhen alle Rechtsvorstellungen des Arbeiters wie des Kapitalisten; alle Mystifikationen der kapitalistischen Produktionsweise" (30).

Marx und Engels zeigten, daß in letzter Instanz, durch alle historischen Zufälligkeiten hindurch die Produktion und Reproduktion des wirklichen Lebens alle Verhältnisse einer Gesellschaft prägt. Je mehr nach und nach alle Produktionsbereiche reell unter das Kapitał subsumiert werden, desto größer ist die Durchschlagskraft des kapitalistischen Reproduktionsprozesses auf alle Lebensbereiche. 
Engels beschreibt im „Ursprung der Familie ... “ dagegen die Fortschrittsmomente, die der Kapitalismus in die heterosexuellen Beziehungen der Proletarier hineinbringt:

„Wirkliche Regel im Verhältnis zur Frau wird die Geschlechtsliebe und kann es nur werden unter den unterdrückten Klassen, also heutzutage im Proletariat - ob dies Verhältnis nun ein offiziell konzessioniertes ist oder nicht. Hier sind aber auch alle Grundlagen der klassischen Monogamie beseitigt. Hier fehlt alles Eigentum, zu dessen Bewahrung und Vererbung ja gerade die Monogamie und die Männerherrschaft geschaffen wurde, und hier fehlt damit auch der Antrieb, die Männerherrschaft geltend zu machen ... Und vollends seitdem die große Industrie die Frau aus dem Hause auf den Arbeitsmarkt und in die Fabrik versetzt hat und sie oft genug zur Ernährerin der Familie macht, ist dem letzten Rest der Männerherrschaft in der Proletarierwohnung aller Boden entzogen - es sei denn etwa noch ein Stück der seit Einführung der Monogamie eingerissenen Brutalität gegen Frauen" (31).

Diese Fortschrittsmomente können sich jedoch nur, wenn überhaupt, gegen eine Tendenz durchsetzen, die letztlich auf das Extrem ,,der erzwungenen Familienlosigkeit der Proletarier" (32) abzielt.

Zwar wird in dem Maße wie die große Industrie die Frau aus dem Haus auf den Arbeitsmarkt und in die Fabrik versetzt hat, tatsächlich -- als eine dem Kapitalismus immanenten Entwicklung - „dem letzten Rest der Männerherrschaft in der Proletarierwohnung aller Boden entzogen", aber andererseits wirkt die ökonomische Charaktermaske des freien und gleichberechtigten Warenbesitzers, die den Lohnarbeitern und -arbeiterinnen mehr und mehr zur zweiten Natur wird, neben der Erfahrung der materiellen Unsicherheit und des Zwangs zur Unterordnung unter die Despotie des Kapitals im Arbeitsprozeß tendenziell zerstörerisch auf die Beziehungen der Geschlechtsliebe und damit auf die Familie. Galt schon für alle Klassengesellschaften, daß ,die Familienordnung ganz von der Eigentumsordnung beherrscht wird" (33), so gilt dies allzumal für den Kapitalismus. Im Zuge der Beseitigung der auf ökonomischer Abhängigkeit der Frau vom Mann gegründeten Männerherrschaft entfaltet sich im Kapitalismus eben nicht ungebrochen die individuelle Geschlechtsliebe. Sie ist im Kapitalismus immer mehr oder weniger von den Warenund Klassenbeziehungen gezeichnet, und wird durch die sich zunehmend auch auf die vermeintlich privatesten Beziehungen destruktiv auswirkenden, in der Zirkulation eingeübten Verhaltensweisen beeinträchtigt. Dauerhaftigkeit und Intensität der Beziehungen, die durch die Herrschaftsstellung des Mannes bislang abgestützt werden, werden deshalb zunehmend bis hin zu Formen der fast völligen Beziehungsunfähigkeit eingeschränkt.

Der bürgerliche Staat versucht der zunehmenden Dysfunktionalität der Kleinfamilie für die Aufzucht des Arbeiternachwuchses u. a. dadurch Rechnung zu tragen, daß er immer mehr ursprünglich der Familie zukommende Familienfunktionen übernimmt.

Die reaktionäre Ideologie, wonach die Familie die Keimzelle des Volkes sei

MEW 21, S. $73 \mathrm{f}$.

32 MEW 4, S. 478

33 MEW 21, S. 28 
usw., trifft immer weniger mit fortschreitender kapitalistischer Entwicklung die Wirklichkeit.

„Die gegenwärtige typische Kleinfamilie stellt somit den kurzlebigen Rest eines einstmals politisch und ökonomisch unabhängigen sozialen Gebildes dar", schreibt Petra Millhofer in ihrer Untersuchung über die Familie im gegenwärtigen Kapitalismus der BRD (34). Milhoffer versucht nichtsdestoweniger in einer „politisch-ökonomischen Funktionsbestimmung der Familie im entfal teten Kapitalismus" (35) zu beweisen, daß ,die Atomisierung der Gesellschaft in politisch und ökonomisch eigenständige ,Privatpersonen " an der familialen Reproduktionsform spezifische Grenzen finden muß" (36). Zwar erwähnt sie in ihrer Beweisführung einmal ,die klassenspezifische Funktion der Familie“ (37), diese reduziert sich für Petra Milhoffer jedoch auf ,allenfalls quantitative Verschiedenheiten der Revenue“ (38). Weder akzeptiert sie die Konsequenzen, die Marx und Engels aus der Klassengeprägtheit der Familie im Kapitalismus zogen, noch setzt sie sich kritisch mit ihnen auseinander. Für Marx und Engels bestand ein fundamentaler Unterschied zwischen der Familie des Bourgeois und den Formen der individuellen Reproduktion in der Arbeiterklasse. In Bezug auf die Bourgeoisie sahen Marx und Engels die Familie als ein für die Vererbung des Privateigentums notwendiges Verhältnis an:

„Die Ehe, das Eigentum, die Familie bleiben theoretisch unangetastet, weil sie praktisch die Grundiagen sind, auf denen die Bourgeoisie ihre Herrschaft errichtet, weil sie in ihrer Bourgeoisform die Bedingungen sind, die den Bourgeois zum Bourgeois machen" (39).

Sahen sie also, daß für die Bourgeoisie deren Familie notwendige Funktionen erfüllt, so galt für das Proletariat, daß ,die Familie wirklich aufgelöst ist" (40).

Es geht nicht an, im Begriff der bürgerlichen Familie derart von historischen und gesellschaftlichen Zusammenhängen zu abstrahieren, daß nur noch ,ein durch Arbeitsteilung zwischen Mann und Frau bestimmter naturwüchsiger Zusammenschluß zur Erhaltung menschlichen Lebens" (41) übrig bleibt. Denn bei einer solchen Abstraktion gerät die Behauptung, daß „der familiale Privathaushalt nach wie vor institutioneller Grundpfeiler des bürgerlich-kapitalistischen Gesellschaftssystems" (42) bleibt, zur Trivialität: um Arbeitskräfte ausbeuten zu können, müssen sie tatsächlich erst einmal gezeugt, geboren und sozialisiert werden. Der historische Formwandel ist damit aber hinwegabstrahiert.

Auch die Feststellung, daß die bürgerliche Kleinfamilie „,bisher noch die billigste Form [ist], um den natürlichen Voraussetzungen der Produktion menschlichen

Petra Milhoffer: Familie und Klasse, Frankfurt a. M. 1973, S. 65

a. a. O., S. 61-78

a. a. O., S. 72

a. a. O., S. 68

a. a. O., S. 70

MEW 3, S. 164

a. a. O., (Hervorh. von Marx und Engels)

Milhoffer, a. a. O., S. 70

a. a. O., S. 76 
Lebens Rechnung zu tragen" (43), ändert nichts an dem Dilemma des gegenwärtigen Kapitalismus, nämlich, „daß die strukturell und funktional zunehmend reduzierte Familie tendenziell dysfunktional zu werden droht" (44). Diesen Sachverhalt benennt Milhoffer zwar treffend, vermittelt ihn aber nicht mit ihrer These von der Familie als dem ,wesentlichen“ Rahmen der individuellen Reproduktion der Arbeiterklasse.

Die Analyse der Tendenz zur Dysfunktionalität der Familie, die etwa gleichzeitig mit der Tendenz zur Integration homosexuellen Verhal tens in die bürgerliche Moral abläuft, sollte nicht durch dogmatische Setzungen von der ,wesentlichen" Funktion der Familie auch in der Arbeiterklasse erschwert werden, ähnlich wie auch die These von der dem Kapitalismus ,wesentlichen" Homosexuellenfeindlichkeit oft allzu voreilig akzeptiert wird. Konsequenterweise müßten dann nämlich auch so merkwürdige Slogans wie „Zerschlagt die Kleinfamilie!“ und „Schwulsein ist eine Waffe im Klassenkampf!" ebenfalls akzeptiert werden. Nicht die Kleinfamilie, sondern die „,atomistische Privatperson“, die auch in ihrem Sexualverhalten von den Gesetzen der Warenbesitzerkonkurrenz beherrscht wird, ist die kleinste Einheit einer Gesellschaft, die mehr und mehr die Reste früherer ökonomischer Zustände beseitigt und immer vollständiger ihrem Begriff entspricht (45). Erst in dem Maße, wie sich Klassenbewußtsein und Solidarität in der Arbeiterklasse entwickeln, entsteht die Grundlage für neue sozialistische Beziehungen zwischen den für die soziale Emanzipation Kämpfenden:

\footnotetext{
„Der Klassenkampf ist für die Arbeiterklasse zugleich eine Schule der Liebe. Ambivalente Gefühlseinstellungen, die soziale Kontakte unterbinden, erfahren eine rationale Aufhebung, wenn die Aggression dem Unterdrücker gelten kann und die Liebe einen Adressaten im Kampfgenossen zu finden vermag... Die emanzipierte Freisetzung der Homosexualität bildet zugleich die Voraussetzung für die Freisetzung von heterosexueller Liebe. Angstfreie libidinöse Bindungen ans andere Geschlecht sind nur möglich, wenn eigene Persönlichkeitsanteile akzeptiert werden können, die dem andern Geschlecht zugerechnet werden und dadurch die Kluft zwischen Mann und Frau überwunden werden kann, ohne daß die Differenz negiert zu werden braucht, an die die Lust gebunden ist" (46).
}

45 Zu den inzwischen von der bürgerlichen Psychiatrie registrierten neueren Auswirkungen dieser Entwicklung auf das Individuum vgl. z. B. R. C. Calogeras und F. X. Schupper, ,Verschiebung* der Abwehrformen und einige Konsequenzen für die analytische Arbeit, in: Gruppendynamik und der, subjektive Faktor', hrsg. von K. Horn, Frankfurt a. M. 1972, S. 312-348. Die beiden New Yorker Psychiater können jedoch die von ihnen konstatierte zunehmende Häufigkeit von zwischenmenschliche Beziehungen verhindernden Charakterstörungen nur kulturpessemistisch als Ergebnisse ,unserer hemmungslos expandierenden industriell-technologischen Gesellschaft" begreifen (vgl. S. 313). Bezeichnend für ihren freudianisch bornierten Standpunkt ist es, wenn sie den „Einstellungswandel gegenüber der Homosexualität" nur als „,Regression“verstehen können, die sich als typisch für eine Gesellschaft erweise, ,die täglich mehr atomosiert wird" (vgl. S. $319 \mathrm{ff}$.). Gerhard Vinnai: Sozialpsychologie der Arbeiterklasse, Reinbek 1973, S. 153 f. 
Dieser Exkurs sollte Momente aufzeigen, die dafür sprechen, daß eine Bedrohung und Zerrüttung heterosexueller Liebesbeziehungen im ernstzunehmenden Maß nur vom kapitalistischen Wirtschaftsprozeß als der in letzter Instanz alle sozialen Beziehungen prägenden Basis ausgeht und $\mathrm{da} B$ die Vorstellung von der die Heterosexualität bedrohenden Homosexualität eine von antihomosexualen Mythen genährte Fiktion ist. Möglicherweise kann diese Fiktion von der bürgerlichen Propaganda künftig wieder als Aufhänger für Sündenbock-Projektionen benutzt werden, etwa nach dem Muster, daß Akzeptierung homosexuellen Verhaltens die vor aller Augen sich abspielende massenhafte Zerriuttung heterosexueller Dauerbeziehungen in Ehe und Familie verursacht habe (47).

\section{Integration der Homosexualität}

Das Christentum erfiilt im Kapitalismus besondere Funktionen und kann nicht vollständig als Relikt des feudalen Überbaus erklärt werden. Als Grundlage für den Fortbestand der Religion im Kapitalismus benennt Engels im „Antidühring“, ,,daß in der heutigen bürgerlichen Gesellschaft die Menschen von den von ihnen selbst produzierten Produktionsmitteln wie von einer fremden Macht beherrscht werden. Die tatsächliche Grundlage der religiösen Reflexaktion dauert also fort und mit ihr der religiöse Reflex selbst" (48). Für die bürgerliche Gesellschaft ist das Christentum zudem die „entsprechendste Religionsform“ (49).

Ähnlich wie das Verbot der Abtreibung oder der Ehescheidung ist sicher auch die in der Bibel verschiedentlich erhobene Forderung nach Homosexuellenunterdrückung keine zentrale Doktrin des Christentums. Dennoch ist in diesen Fragen die kirchliche Propaganda ein wichtiges Fortschrittshindernis. Es spricht jedoch einiges dafür, daß sich in der weder die Kapitalherrschaft noch die kirchliche Autorität zentral berührenden Frage der Homosexualität wissenschaftliche Erkenntnisse gegen religiöse Dogmen durchsetzen lassen.

Außerdem werden spezifische Konsumbedürfnisse der Homosexuellen und die mehr und mehr enttabuisierten homosexuellen Bedürfnisse der „Normalen“ zunehmend als profitträchtige Kapitalanlagesphären erkannt. Dies schreitet in dem Maße fort, wie sich die Homosexuellen genau so „frei“ entfalten können wie die anderen Konsumentenmassen. Ansätze zur Entwicklung eines Marktes für homosexuelle

47 Derartige Tendenzen deuteten sich etwa an in der Berichterstattung der Bild-Zeitung zum Ihns-Prozeß im September 1974. In einer großen Kampagne wurde versucht, den Eindruck zu erwecken, als sei die homosexuelle Beziehung der beiden angeklagten Frauen die zwangsläufige Ursache für die zerrïttete Ehe der einen Angeklagten und schließlich sogar für die Ermordung des Ehemanns. Hauptgrund dürfte jedoch für diese Berichterstattung der Bild-Zeitung und anderer bürgerlicher Zeitungen im pornographischen Effekt liegen, den für männliche heterosexuelle Leser eine Schilderung weiblicher Homosexualität, noch dazu mit einer Mordstory verquickt, erbringen kann und der hier vor allem ausgenutzt wurde.

48 MEW 20, S. 295

49 MEW 23, S. 93, vgl. auch MEW 26.3, S. 442, wo Marx das Christentum als die ,spezielle Religion des Kapitals" bezeichnet. 
Käufer lassen sich heute in der homosexuellen Subkultur ebenso nachweisen wie die Ausbeutung einer zunehmend enttabuisierten Homosexualität durch die Unterhaltungsindustrie (Film, Showbusiness) und die Textilmodebranche.

Eines der auffälligsten Resultate für die Tendenz zur Nivellierung der zusätzlichen Homosexuellenunterdrückung ist wohl in der Lockerung der antihomosexuellen Sondergesetze in einer Reihe von kapitalistischen Ländern zu sehen (Großbritannien, BRD, Dänemark, Schweden, Niederlande, Australien; in den USA wurde seit 1961 in acht Staaten die Straffreiheit für Homosexualität unter Erwachsenen eingeführt). Eine völlige rechtliche Gleichstellung ist jedoch durch keine dieser neueren Reformen verwirklicht worden (50). Der Prozeß, auf den hier hingewiesen werden soll, nämlich die Aufhebung der zusätzlichen Homosexuellenunterdrückung, ist also noch lange nicht abgeschlossen, wie nicht zuletzt der Stand der Strafrechtsentwicklung beweist. Die diesen Prozeß in Zukunft charakterisierenden Formen, die Geschwindigkeit (51) und die in ihm eingeschlossenen möglichen Rückschläge können im vorliegenden Zusammenhang nicht prognostiziert werden.

Als Marx in Bezug auf die bürgerliche Gesellschaft konstatierte, daß aus untergegangenen Gesellschaftsformen stammende und ,,teils noch unüberwundene Reste sich in ihr fortschleppen" (52), da war ihm klar, daß über Geschwindigkeit und konkreten Verlauf des Abbaus dieser Reste keine allgemeine Aussage möglich ist. Daß diese „Reste" früher oder später verschwinden müssen, war für Marx und Engels jedoch eindeutig. Im Kommunistischen Manifest heißt es über die bürgerliche Gesellschaft:

50 Mit der Tatsache, daß in Frankreich, Spanien und Belgien eine gewisse Zunahme der Homosexuellenunterdrückung durch den Staat zu verzeichnen ist, versucht G. Hocquenghem in seinem Buch „Das homosexuelle Verlangen“ (München 1974) eine allgemeine Zunahme der ,antihomosexuellen Paranoia“ im Kapitalismus zu beweisen (S. 26 ff.). Dagegen spricht jedoch schon, daß in viel mehr Ländern das Strafrecht sich genau entgegengesetzt entwickelt. Hocquenghem braucht seine Behauptung obendrein für einen fragwürdigen Zweck: Homosexuelle werden von ihm zu Märtyrern und Avantgardisten kommender gesellschaftlicher Umwälzungen hochgejubelt, Er wendet sich gegen ,das traditionell-revolutionäre Denken und Handeln“ (S. 134) und glaubt, daßß ,der homosexuelle Kampf" besonders der Pariser Homosexuellengruppe FHAR ein „,neues revolutionäres Modell“ (S. 133) zu entwickeln imstande sei. Das Ziel ist, in Anlehnung an Fourier, die Abschaffung der ,Zivilisation“ (S. 135 f.).

51 Daß z. B. die Gleichberechtigung der Frau ähnlich wie die der Homosexuellen, trotz aller Fortschritte, noch immer weit von einer Verwirklichung entfernt ist, weist auf die Langwierigkeit solcher Prozesse hin. Frauenunterdrückung gab es ähnlich wie Homosexuellenunterdrückung auch schon in vorkapitalistischen Gesellschaftsformationen. Je weiter der Ursprung eines Überbaubestandteils in der Menschheitsgeschichte zurückliegt, desto langwieriger scheint der Prozeß seines Abbaus zu sein. Hat er unter gewissen Umständen, wie gegenwärtig die Diskriminierung der Frauen und Homosexuellen, sogar noch einen Nutzeffekt für die Stabilisierung der bestehenden Produktionsweise (bei den Frauen: Lohndrücken; bei den Homosexuellen: Ablenkungs- und „Sündenbock"-Funktionen; in beiden Fällen auch noch Stützung der christlichen Ideologie), so erhöht dies seine Abbauresistenz.

52 Karl Marx, Grundrisse S. 26; vgl. auch MEW 3, S. 312, wo sich Marx und Engels über die Fortvegetation veralteter Rechtsnormen äußern: „Es ist möglich, daß Rechte und Gesetze sich ,forterben', aber sie sind dann auch nicht meht herrschend, sondern nominell, wovon die altrömische und englische Rechtsgeschichte eklatante Beispiele liefern." 
„Alle festen eingerosteten Verhältnisse mit ihrem Gefolge von altehrwürdigen Vorstellungen und Anschauungen werden aufgelöst, alle neugebildeten veralten, ehe sie verknöchern können. Alles Ständische und Stehende verdampft, alles Heilige wird entweiht, und die Menschen sind endlich gezwungen, ihre Lebensstellung, ihre gegenseitigen Beziehungen mit nüchternen Augen anzusehen" (53).

Wichtig ist für uns zunächst der Nachweis einer Tendenz zur Homosexuellenintegration sowie die Überlegung, daß diese Tendenz der Logik des Kapitals entspricht, allen formal das gleiche Recht zukommen zu lassen und alle Unterschiede auf den einen zu reduzieren, den Gegensatz zwischen Arbeiter- und Kapitalistenklasse. „Die ganze Gesellschaft spaltet sich mehr und mehr in zwei große feindliche Lager, in zwei große, einander direkt gegenüberstehende Klassen: Bourgeoisie und Proletariat" (54).

Einerseits ist mit der fortschreitenden Entwicklung der bürgerlichen Gesellschaft, mit der Beseitigung vorkapitalistischer Relikte die Tendenz zur Integration der Homosexuellen in die bürgerliche Gesellschaft festzustellen, denn eine Gefährdung des im Kapitalismus herrschenden Interesses an Unterdrückung und Ausbeutung der Arbeiterklasse ist mit der gesellschaftlichen Akzeptierung homosexuellen Verhaltens nicht verbunden; sowohl als ausbeutbare Arbeitskraft als auch als gute Konsumenten sind Homosexuelle genau so brauchbar wie andere, und diese Brauchbarkeit wird durch besondere Homosexuellenrepression nicht gefördert, sondern behindert.

Andererseits wirkt die von christlich-jüdischer Religion geprägte Sexualmoral zusammen mit besonders in Krisenzeiten anzutreffenden sozialpsychologischen Mechanismen (Konkurrenzdruck erzeugt $\mathrm{Haß}$ auf abweichendes Verhalten, Sündenbockprojektionen usw.) hemmend auf diese Tendenz. Wollte man nachweisen, daß diese hemmenden Momente im Kapitalismus letztlich unüberwindlich sind und daß die Unterdruickung der Homosexuellen nicht dem jeweiligen allgemeinen Maß der Unterdrückung angeglichen werden kann, dann müßte der genaue Punkt angegeben werden, über den die Integration der Homosexuellen nicht hinausgehen kann, ohne den Kapitalismus zu bedrohen. Eine offene Frage, die zur vollständigen Funktionsbestimmung der Homosexuellenunterdrückung im Kapitalismus zu beantworten ist, lautet demnach: Gibt es psychologische und speziell sozialpsychologische Gesetzmäßigkeiten, die für das Verhalten der Menschen im Kapitalismus dahingehend wirken, daß sie notwendigerweise die Unterdrückung der Homosexuellen bedingen?

Von der Beantwortung dieser Frage nach einem womöglich systembedrohenden Charakter der Homosexualität hängt unmittelbar die Einschätzung der Reichweite des homosexuellen Emanzipationskampfes ab: Hat dieser Kampf an und für sich schon revolutionäre Momente in sich, indem er z. B. eine für den Kapitalismus konstitutive Geschlechterrollenverteilung untergräbt, die die Herrschaft des Mannes über die Frau beinhaltet? Oder hat dieser Kampf, gleichgültig, für wie ,revolutionär" die darin engagierten Homosexuellen sich selber halten, allenfalls die Funktion, die längst fällig Subsumtion dieses Bereichs der Sexualität unter die kapitalisti-

54 MEW 4, S. 463 
schen Bedingungen zu beschleunigen? Wäre letzteres der Fall, dann würde das antikapitalistische Moment dieses vermeintlich auf ,sexuelle Revolution“ abzielenden Kampfes in seiner Überleitungsfunktion bestehen, d. h. daß durch die Beseitigung der Schranken, die die Homosexuellen zur Absonderung in eine gettoartige Subkultur und in die Vereinzelung zwangen, die Möglichkeit geschaffen wird, daß die Homosexuellen sich in relevantem Ausmaß ihrer wesentlichen Unterdrückung - nämlich der als ausgebeutete Lohnarbeiter - bewußt werden können und daraus politische Konsequenzen ziehen.

Wenn wir hier der zweiten Alternative zuneigen, dann sind wir uns, wie gesagt, des hypothetischen Charakters dieser Einschätzung bewußt. Es ist nicht völlig ausgeschlossen, daß sozialpsychologische Gese tzmäßigkeiten existieren könnten, die eine andere Einschätzung erfordern. Nach den vorliegenden Erkenntnissen erscheint es uns jedoch plausibler, die noch nicht verwirklichte Angleichung der Homosexuellenunterdrückung an die allgemeine nicht der antihomosexuellen Tendenz des Kapitalismus zuzuschreiben, sondern vielmehr der noch nicht vollständigen Beseitigung vorkapitalistischer Relikte durch das Kapital selbst.

Wer dem derzeitigen Kapitalismus nur noch „Stagnation und Fäulnis“ und "Reaktion auf der ganzenLinie" zubilligt, für den wird der zunehmende Abbau der Homosexuellendiskriminierung ein völliges Mysterium bleiben, wenn er nicht Homosexualität sowieso schon immer als Verfallserscheinung des dekadenten Spätkapitalismus verstanden hat. Von einem Standpunkt aus, bei dem zur Frage der Homosexualität noch Angst und Mythologie, Dekadenz- und Entartungstheorien die sexualwissenschaftliche Aufklärung überschatten, ist die Konstruktion eines Zusammenhangs zwischen ,unsauberem" Sexualleben und der Fäulnis der herrschenden Klasse eine naheliegende Vorstellung. Die Haltung der SPD zur Krupp-Affäre 1902 wurde bereits erwähnt, die Große Sowjetenzyklopädie aus dem Jahre 1954 bringt jene Vorstellung in klassischer Formulierung zum Ausd ruck:

„Homosexualität ist seit dem hohen Altertum bekannt. Schon die Sklavenhaltergesellschaft schuf infolge der Übersättigung und Perversität der herrschenden Klassen günstige Bedingungen für Laster und Widernatürlichkeit. In der kapitalistischen Gesellschaft ist Homosexualität eine verbreitete Erscheinung. Es genügt, auf die Existenz homosexueller Prostitution in den kapitalistischen Ländern hinzuweisen ... In der sowjetischen Gesellschaft mit ihrer gesunden Moral gilt die Homosexualität als sexuelle Perversion, als schädlich und kriminell ... “ (55).

\section{Sexualwissenschaft und Vorurteil}

Engels beschreibt die allgemeine Verlaufsform von Prozessen der Überwindung altertiümlicher Ideologien bekanntlich folgendermaßen:

„Was nun die noch höher in der Luft schwebenden ideologischen Gebiete angeht, Religion, Philosophie etc., so haben diese einen vorgeschichtlichen Bestand von was wir heute Blödsinn

55 Große Sowjetenzyklopädie (Bol’ǎja Sovetskaja Éciklopedija), Moskva 1954, Bd. 12, S. 35 (Übers. d. Verf.) 
nennen würden. Diesen verschiedenen falschen Vorstellungen von der Natur, von der Beschaffenheit des Menschen selbst, von Geistern, Zauberkräften etc. liegt meist nur negativ Ökonomisches zugrunde; die niedrige ökonomische Entwicklung der vorgeschichtlichen Periode hat zur Ergänzung, aber auch stellenweise zur Bedingung und selbst Ursache, die falschen Vorstellungen von der Natur. Und wenn auch das ökonomische Bedürfnis die Haupttriebfeder der fortschreitenden Naturerkenntnis war und immer mehr geworden ist, so wäre es doch pedantisch, wollte man für all diesen urzuständlichen Blödsinn ökonomische Ursachen suchen. Die Geschichte der Wissenschaft ist die Geschichte der allmählichen Beseitigung dieses Blödsinns, resp. seiner Ersetzung durch immer weniger absurden Blödsinn ${ }^{\text {(5 }}$ (56).

Die Ende des 19. Jahrhunderts sich aus der Medizin und speziell aus der Psychiatrie entwickelnde sexualwissenschaftliche Erforschung der Homosexualität kann als treffendes Beispiel dieser Engelsschen Darlegung angesehen werden. Die von H. Ellis in England und von R. von Krafft-Ebing in Österreich begonnene systematische Erforschung der Homosexualität führte zunächst zu dem Ergebnis, daß es sich hierbei um eine Krankheit handele und deshalb nicht strafrech tlich verfolgt werden dürfe (57). M. Hirschfeld ging mit seiner Theorie, wonach Homosexualität einer „angeborenen" sexuellen Zwischenstufe zwischen Mann und Frau zuzuordnen sei, immerhin als erster von der Auffassung ab, daß Homosexualität eine Krankheit sei (58).

Daß auch heute in der bürgerlichen Sexualwissenschaft zur Frage, ob Homosexualität eine Krankheit sei, sich noch nicht voll eine angemessene Auffassung durchgesetzt hat, wird deutlich an einem äußerst markanten Wendepunkt in dieser Diskussion: Auf der Jahrestagung der American Psychiatric Association wurde am 9.5. 1973 beschlossen, Homosexualität aus der Liste der Krankheiten zu streichen (59). Daß „die Geschichte der allmählichen Beseitigung dieses Blödsinns“, der Beseitigung der Krankheitsdefinition der Homosexualität, eine noch lange nicht abgeschlossene, auch die Möglichkeit von Rückschlägen einschließende Entwicklung darstelit, wird deutlich an der Ankündigung des führenden Vertreters der Krankheitsanschauung, Socarides (60), sich nicht mit der Entscheidung der Psychiatergesellschaft abzufinden und sich für die Revision des Beschlusses einzusetzen.

Unter den Anhängern der Krankheitsanschauung nehmen die Frankfurter Freudianer R. Reiche und M. Dannecker eine interessante Zwischenposition ein. Auch für sie ist die Homosexualität eine ,schwere Stönung“ (61). Dem entspricht ihrer Ansicht nach jedoch auf der anderen Seite eine pathologische Formung der „normalen Heterosexualität":

56 MEW 37, S. 492, Engels' Brief̂ an C. Schmidt vom 27.10.1890

57 Vgl. hierzu z. B. R. v. Krafft-Ebing, Der Conträrsexuale vor dem Strafrichter, 2. Aufi., Wien 1895

58 Magnus Hirschfeld, Die Homosexualität des Marınes und des Weibes, Berlin 1914

59 Bericht vom Meeting der American Psychiatric Association, in: American Journal of Psychiatry 130:11, Nov. 1973

60 C. W. Socarides ist in der BRD durch sein Buch, ,Der offen Homosexuelle" (Frankfurt a. M. 1971) bekannt. Vgl dazu die treffende Kritik von J. Hempel in: Das Argument, Jg: 14, 172, Heft 5/6, S. $838 \mathrm{II}$.

61 M. Dannecker, R. Reiche, Die kollektive Neurose der Homosexuellen, in: Leviathan, Zeitschrift für Sozialwissenschaft, Heft 1, 197, S. 63 
„Man kann die Pathologie der Homosexuellen nicht aus ihrer Heterosexualitätsangst begründen, ohne sich zugleich über die Homosexualitäts-Angst der Heterosexuellen Rechenschaft zu geben. Für die kollektiv herrschende Integrationsform der Triebe und der Objektbeziehungen, die in unserer Kultur als ,normale Heterosexualität" gilt, ist die Verdrängung der homosexuellen Strebungen konstitutiv und, als Konsequenz daraus, die unbewußte Angst vor der Homosexualität. Die kulturell vorherrschende Form der Heterosexualität ist notwendig pathologisch" (62).

Der in diesem Gedanken enthaltene Hinweis auf die spätestens seit S. Freuds „,Drei Abhandlungen zur Sexualtheorie" bekannte bisexuelle Beschaffenheit aller Menschen wird zweifellos für eine auch die Psychologie umfassende Untersuchung über die Gründe der Homosexuellenunterdrückung von großer Bedeutung sein (63). Die mehr oder weniger bewußte Angst vor den nur mangelhaft unterdrückten homosexuellen Wünschen ist sicher beteiligt am Zustandekommen von homosexualitätsfeindlichen Einstellungen bei „,normalen Heterosexuellen“. Wenn Dannecker und Reiche ihrem Befund jedoch ohne weiteres Gültigkeit für die gesamte ,gegenwärtige gesellschaftliche Epoche" zusprechen (64), dann zeigt sich schon hier, trotz ihrer verschiedentlichen Berufung auf Karl Marx, ihre Kurzsichtigkeit gegenüber realen gesellschaftlichen Veränderungen.

Eine immer noch ausstehende materialistische Analyse der Formen und Funktionen von Sexualunterdrückung in der bürgerlichen Gesellschaft darf nicht bei der abstrakten Versichenung stehen bleiben, daß der Kapitalismus die Sexualität der Menschen unterdrückt, daß diese Unterdrückung von der Arbeitswelt ausgehend über familiale Instanzen vermittelt schon in der frühkindlichen Sozialisation der Individuen beginnt, daß dadurch zwanghaft fixierte ,normale“ Heterosexualität in Zwangshomosexualität ihre Ergänzung findet usw. Auch die pauschale Behauptung, daß ,zum Konstitutionsprozeß kulturell normaler Heterosexualität [...] unabdingbar kollektiv-neurotische Elemente [ . . gehören, die] unabdingbar für den gesellschaftlichen Funktionsżusammenhang, mindestens für den der gegenwärtigen kapitalistischen Gesellschaften sind"(65), tragen schon wegen ihrer Abstraktheit nichts zur Lösung dieser Aufgabe bei.

Eine Analyse muß vielmehr, ausgehend von der allgemeinen Begrifflichkeit des wissenschaftlichen Sozialismus, aus den Entwicklungsstufen der bürgerlichen Gesellschaft in ihrer jeweils historisch-konkreten Ausprägung die vorhandenen Formen der Sexualunterdrückung erklären. Von diesen, durch den Entwicklungsprozeß der Produktivkräfte und des Klassenkampfs geprägten Form- und Funktionsveränderung zu abstrahieren und die Phänomene der erscheinenden Oberfläche nur als beständige Wiederkehr der ewig gleichen Sexualunterdrückung zu begreifen, führt 62 a. a. O., S. $63 \mathrm{f}$.

63 Vgl. auch W. F. Haug, Warenästhetik Sexualität und Herrschaft, (Frankfurt a. M. 1972), S. 172: „Die Unterdrückung besonderer Formen der Sexualität zielt m. E. immer auf den ganzen Umfang und Anspruch des Triebes. Die bürgerlichen Produktionsverhältnisse bewirken und verlangen eine durch völlige Brechung ihrer Spontaneität und feste Kanalisierung ihrer Unbestimmtheit erkaufte Domestizierung des Sexuellen. So ist nicht in erster Linie die zwanghafte Homosexualität, sondern die ,Zwangsheterosexualität ${ }^{*}$ (Ferenczi) zu analysieren."

M. Dannecker, R. Reiche, a. a. O., S. 65

65

a. a. O., S. 66 
zu dogmatischen und praxisfernen Schlußfolgerungen. Und auch das entgegengesetzte Extrem, die empiristische Vorgehensweise macht blind für die Schranken, die der Kapitalismus der sexuellen Emanzipation setzt.

Dannecker und Reiche sehen in den Ergebnissen einer Befragung von 789 homosexuellen Männern (hohe Rate von Selbstmordversuchen, verbreiteter Wunsch nach „Behandlung“ der eigenen Homosexualität, Verachten homosexuellen effiminierten Verhaltens durch die Homosexuellen selbst usw.) nicht etwa das Resultat der lang andauernden terroristischen Diskriminierung durch Staat und öffentliche Meinung. Stattdessen sehen Dannecker und Reiche in der „Schulmedizin“ und der „klinischen Praxis und theoretischen Literatur der Psychoanalyse“ (66) die angemessenen Kategorien für die Interpretation ihrer Befunde vorgegeben. Vom Standpunkt des Arztes aus sind die Ergebnisse der Befragung lediglich Symptome der ,kollektiven Neurose der Homosexuellen“ als „Schutz gegen offen neurotische Erkrankungen“(67). So wird z. B. auch ,militanter Antikommunismus“ in der Dimension normal - pathologisch begriffen (68). In der Einschätzung der homosexuellen Männer als „,kollektiv-neurotisch“ sehen Dannecker und Reiche ihren Beitrag, um die „psychologische Seite der Ideologiekritik“ herauszuarbeiten (70). Sie scheinen zu glauben, daß eine ,materialistische Psychologie“ hauptsächlich die Resultate der Klassenanalyse und die Erscheinungsformen des Klassenkampfs in einer psychoanalytischen Fachsprache neu zu beschreiben habe. Sie verraten aber nicht, welchen Sinn es haben soll, Stufen des Klassenbewußtseins und der Einsicht in die Bedingungen der Emanzipation in Begriffe wie „pathologisch“ und „normal“, krank und gesund zu fassen. Es sei denn, Sozialisten könnten ihre Funktion im Klassenkampf besser erfüllen, wenn sie gleichsam ausgerüstet mit dem autoritären Image von Ärzten der Arbeiterklasse den Weg zur sozialistischen Gesundheit weisen . . (71). So scheint denn der Verdacht nicht unbegründet, daß hier die Freudsche Theorie als Marxismusersatz fungieren soll. Dieser Verdacht drängt sich etwa auch auf, wenn Dannecker und Reiche von Freuds Religionskritik als dem „Kernstück für jede materialistische Religionskritik" (72) sprechen, ohne auch nur den Versuch einer Vermittlung mit den Aussagen des wissenschaftlichen Sozialismus zu dieser

a. a. O., S. 62

a. a. O., S. 66

a. a. O., vgl. S. 65

a. a. $\mathrm{O}$.

a. a. 0 .

70

So grotesk diese Überlegungen auch sein mögen, haben sie doch neuerdings eine gewisse Anhängerschaft gewonnen. M. Schneider beispielsweise vertritt in seinem Bestseller „Neurose und Klassenkampf" (Reinbek 1973) den gleichen Standpunkt: „Beschränkte sich die klassische Psychoanalyse darauf, den Kranken in der Gesellschaft zu behandeln, so ist es Aufgabe einer materialistisch orientierten, emanzipativen Psychoanalyse, die Gesellschaft selber als Krankheit zu behandeln." (S. 10) Homosexuellenunterdrückung existiert für Schneider übrigens nur in der Sowjetunion (vgl. S. 46). Im Kapitalismus hält er die ,gesellschaftliche Blockierung und Tabuisierung der gleichgeschlech tlichen Erotik“" nur im Hinblick auf die „ödipale Rivalität" in der Kleinfamilie und im Hinblick auf Faschisten, deren „Kameraderie usw." aus der „Verdrängung der Homosexualität" resultiert, für erwähnenswert (vgl. S. 76 und 117). Dannecker, Reiche, a. a. O., S. 65 
Frage zu versuchen. Die Bemerkung, Marx habe lediglich die ,ökonomische Seite“ der Ideologiekritik herausgearbeitet (73), zeugt von einem prinzipiellen Unverständnis gegenüber dem wissenschaftlichen Sozialismus und nährt den Verdacht, daß unter dem Anspruch, den Marxismus durch die Psychoanalyse zu ergänzen, dieser durch jene ersetzt werden soll. Nichtsdestoweniger bleibt aber die Erforschung der Tatsachen über die gegenwärtige Lage homosexueller Männer in der BRD, ungeachtet des fragwürdigen theoretischen Rahmens, ein großes Verdienst von Dannecker und Reiche. Für die Gewinnung einer wirksamen Taktik des Emanzipationskampfes der Homosexuellen sind aus ihrer Untersuchung grundlegende empirische Daten zu entnehmen.

In dem vorliegenden Zusammenhang ist es noch erwähnenswert, daß auch Dannecker und Reiche Momente konstatieren, die auf eine Integration der Homosexuellen in die bürgerliche Gesellschaft hindeuten. So stellten sie fest, daß in der Medizin ,unter dem Druck fortschreitender naturwissenschaftlicher und psychologischer Erkenntnisse" (74) die Homosexualität zwar immer noch als Krankheit aufgefaßt wird, jedoch schon ein Begriffswandel eingetreten ist: Homosexualität ist nach herrschender Auffassung nicht mehr eine „Psychopathie“, sondern „Deviation". Weiterhin sind Dannecker und Reiche zwar von dem Vorhandensein einer „gegenwärtigen Toleranzströmung gegenüber Homosexuellen“ überzeugt (75), sehen aber darin kein Phänomen, der der Erklärung bedürftig wäre.

Einen illustrierenden historischen Abriß, der den Wandel in der Einstellung zur Homosexualität und auch die besonderen Entwicklungen in den sozialistischen Ländern, besonders der Sowjetunion und der Volksrepublik China (76) miteinbezieht, kann hier von uns noch nicht vorgelegt werden. Für die Zurückweisung der Ansicht von der ewig gleichbleibenden Haltung gegenüber der Homosexualität in der bürgerlichen Gesellschaft wäre dies jedoch unbedingt erforderlich. Ein solcher Abriß müßte Zusammenhänge zwischen der allgemeinen Einstellung zur Sexualität, wie sie sich etwa im Form- und Funktionswandel der Familie und der Prostitution zeigt, und den verschiedenen Abstufungen in der Einstellung zur Homosexualität beinhalten (77). Die Abschaffung der Todesstrafe gegen Homosexuelle müßte hier ebenso nachgezeichnet werden wie die Vereinnahmung des Themas durch die Psychiatrie und die entstehende Sexualwissenschaft am Ende des 19. Jahrhunderts; die seuchenartige Verbreitung der Syphilis als einer Grundlage der weitgehenden und sich steigernden Sexualitätsfeindlichkeit seit Beginn des 16. Jahrhunderts ebenso wie die nach dem Zweiten Weltkrieg sich verstärkende Enttabuisierungstendenz, die etwa gleichzeitig mit der Veröffentlichung der Kinsey-Berichte einsetzte usw. Erst anhand solcher historischer Studien könnten die hier vorgetragenen Überlegun-

73

74

75

76

77 a. a. $\mathrm{O}$.

a. a. O., S. 62

a. a. O., S. 68

Nach unseren - im einzelnen jedoch nicht genügend abgesicherten - Informationen herrscht in beiden Ländern die schärfste Homosexuellenunterdrückung.

Die umfangreichen Studien von Iwan Bloch werden hierbei von besonderer Wichtigkeit sein: „Das Geschlechtsleben in England“ (Berlin 1903), „Der Marquis de Sade und seine Zeit" (Berlin 1904), „,Der Ursprung der Syphilis" (Jena 1901) u. a. 
gen auf ihre Gültigkeit hin konkret überprüft werden. Es käme darauf an, das umfangreiche vorliegende Tatsachenmaterial zu sichten und für eine materialistische Analyse auszuwerten, zu entscheiden, bis zu welchem Grad Homosexuellenunterdrückung in bestimmten Phasen der Entwicklung der bürgerlichen Gesellschaft wirklich erforderlich war.

\section{Der kleinbürgerlich-demokratische Charakter der homosexuellen Emanzipa- tionsbewegung in Deutschland}

Organisatorischen Ausdruck fand das Emanzipationsbedürfnis der Homosexuellen erstmals im Jahre 1897 in Deutschland (78). In Charlottenburg wurde von dem Arzt Magnus Hirschfeld, dem Verleger Max Spohr und dem Ministerialbeamten Erich Oberg das Wissenschaftlich-humanitäre Komitee (WHK) gegründet. Es umfaßte bald einige tausend Mitglieder und hatte Untergruppen in mehreren deutschen Städten, in Österreich, England und in den Niederlanden.

Die gesellschaftliche Situation jener Zeit war nicht nur gekennzeichnet durch die nach Aufhebung der Sozialistengesetze erstarkende und mehr und mehr in Revisionismus verfallende SPD; zugleich entstand auch eine vielfältige, mehr oder weniger locker mit der Arbeiterbewegung verbundene Lebensreformbewegung. Hirschfelds rückblickende Schilderung der Situation am Ende des 19. Jahrhunderts bietet hierfür eine nützliche Illustration:

„Es ist kein Zufall, daß die Gründung der deutschen Wandervogelbewegung und der ersten Landerziehungsheime in die gleiche kurze Zeitspanne fällt, in der völlig unabhängig voneinander eine ganze Anzahl sexualreformistischer Bewegungen entstand; wir nennen nur die Gesellschaft zur Bekämpfung der Geschlechtskrankheiten, die es wagte, ein Übel beim richtigen Namen zu nennen, das zu erwähnen fast für schlimmer galt, als es zu haben (selbst auf den Krankenkassenscheinen der Arbeiter durften vorher Syphilis und Tripper nicht vermerkt werden, da sie als ,selbstverschuldet" von jeder Hilfeleistung der Versicherung ausgeschlossen waren) - die Mutterschutzbewegung, die sich der unehelichen Mutter und des unehelichen Kindes annahm, die nicht minder als die Geschlechtskranken als Parias der Gesellschaft angesehen wurden - das Wissenschaftlich-humanitäre Komitee, das den Rechtfertigungs- und Verteidigungskampf gegen die gesetzliche und gesellschaftliche Verfolgung gleichgeschlechtlich geborener Menschen aufnahm. Auch die ,Internationale abolitionistische Föderation', welche unter Führung der edlen Josephine Butler sich zunächst in Englang gegen die Kasernierung und Reglementierung der Prostitution wandte [...], griff in jener Zeit auf Deutschland über. Und vor allem traten die damals ,radikal' genannten Vorkämpferinnen der Frauenemanzipation auf den Plan. 1894 fand

Lange Zeit blieb Deutschland das einzige Land, in dem sich die Homosexuellen organisiert zur Wehr setzten. Zum Überblick vor allem über die anderen Länder vgl. die Broschüre von J. Lauritsen und D. Thorstad, "The Homosexual Rights Movement" (New York 1973). Zur Geschichte des WHK vgl. vor allem, ,Jahrbuch für sexueile Zwischenstufen", Leipzig und Berlin 1.1899-23.1923. Ferner: Ralf Seidel, Sexologie als positive Wissenschaft und sozialer Anspruch, Med. Diss. München 1969; und Jim Steakley: The gay movement in Germany. In: The Body Politic, Toronto 1973/74, Nr. 9, 10, 11 und 13 
in Berlin zum nicht geringen Erstaunen und Entsetzen aller bürgerlichen Parteien die erste größere Kundgebung für das Frauenstimmrecht statt, der 1896 der erste große ,Internationale Frauenkongreß für Frauenwerke und Frauenbestrebungen' folgte . . . Bezeichnend für den Zug der Zeit war es auch, daß alle Bestrebungen für naturgemäße Lebens- und Heilweise in den letzten Jahrzehnten des 19. Jahrhunderts sehr im Volke an Verbreitung gewannen; so wurde 1896 in Berlin die erste Loge gegen den Alkohol gegründet, der viele weitere folgten [ . . . ] Ich selbst nahm besonders nach meiner Niederlassung in Berlin (am 1.5.1896) an allen diesen Bestrebungen regen Anteil“" (79).

Die Klassenlage der drei Gründer des WHK steht in engem Zusammenhang mit dem kleinbürgerlich-demokratischen Charakter der Politik dieser Organisation. Die wichtigste Aktivität bestand zunächst darin, eine von Hirschfeld verfaßte Petition an den Reichtstag zur ersatzlosen Streichung des $\$ 175$ in den ,höheren Kreisen der Gesellschaft" $z u$ verbreiten und für Unterstützung zu werben. Prominente Wissenschaftler, Künstler, Politiker, Literaten, höhere Beamte, Kirchenfunktionäre waren die Adressaten dieser Bemühungen, die auch in der SPD-Spitze nicht ohne Resonanz blieben: Bebel und Kautsky unterzeichneten schon 1898 die Petition (80). Auch die publizistischen Aktivitäten des Komitees richteten sich zunächst ausschließlich an die „höheren Stände"; das vom Komitee herausgegebene Jahrbuch für sexuelle Zwischenstufen enthielt z. B. fast ausschließlich sexualwissenschaftliche Abhandlungen.

Die Arbeiterkiasse kam zunächst einmal als Forschungsobjekt für das seinem Selbstverständnis nach völlig unpolitische und ,auf dem Boden der bestehenden Gesellschaftsordnung" (81) stehende WHK vor: 1904 wurde unter 6000 Mitgliedern des Verbandes deutscher Metallarbeiter eine Umfrage veranstaltet, um zu ermitteln, bei wie vielen Metallarbeitern „der Geschlechtstrieb immer nur auf weibliche, immer nur auf männliche oder sowohl auf weibliche wie auf männliche Personen gerich tet" ist (82).

Nach und nach wurde jedoch auch eine breitere Propaganda- und Aufklärungsarbeit entfal tet, die auf die Gesamtbevölkerung gerichtet war und Vortragsund Diskussionsveranstaltungen in allen größeren deutschen Städten sowie Verbreitung von Aufklärungsschriften umfaßte. Hilfe für Homosexuelle bei Erpressungsfäl-

M. Hirschfeld, Geschlechtskunde, Bd. 1, Stuttgart 1926, S. 377. Es ist bezeichnend für Hirschfelds politischen Standpunkt, daß er den bürgerlichen Charakter des erwähnten Internationalen Frauenkongresses von 1896 verschweigt. Zu der Tatsache, daß Klara Zetkin und zwei andere Genossinnen eingeladen waren, schrieb die sozialdemokratische „Gleichheit": „Es bedarf wohl kaum der Mittheilung, daß die drei Genossinnen höflich aber bestimmt abgelehnt haben, an dem Kongreß theilzunehmen. Sie sind der Ansicht, daß auf einem Kongreß, der ein ausgesprochen bürgerliches Gepräge trägt und auf dem die ,Arbeiterinnenfrage' doch nur so nebenher behandelt werden kann, Sozialdemokratinnen ebenso wenig etwas zu suchen haben, als unsere Genossen auf den Kongressen der Freidenker, bürgerlicher Demokraten und Sozialreformler." (Die Gleichheit, Jg. 6, 1896, S. 144)

80 Vgl. Jahrbuch für sexuelle Zwischenstufen, Jg. 1, 1899, S. 252

81 Monatsbericht des WHK, Jg. 3, 1904, Nr. 11, S. 1, Satzung des WHK.

82 Das Ergebnis der statistischen Untersuchungen über den Prozentsatz der Homosexuellen, in: Jahrbuch für sexuelle Zwischenstufen, Jg. 6, 1904, S. $111 \mathrm{ff}$. Von den 1912 ausgewerteten Antworten waren 9,25\% heterosexuell, 3,19\% bisexuell und 1,15\% homosexuell. 
len und Unterstïtzung vor Gericht bei Strafprozessen wegen $\S 175$ bildeten darüber hinaus einen wichtigen Teil der WHK-Aktivitäten. Die wichtigste Aufklärungsschrift, die den Titel träge ,Was soll das Volk vom dritten Geschlecht wissen? “ wurde von 1901 an bis zum Ersten Weltkrieg in ca. 50000 Exemplaren verbreitet. In der sozialdemokratischen „Neuen Zeit" wurde diese Schrift jedoch treffend als für die Volksaufklärung ungeeignet kritisiert. Statt Vorurteile zu bekämpfen ,,bringt die Broschüre ganze Seiten voll mehr oder weniger bekannter Namen aus der Gegenwart mit mehr oder weniger tönenden Titeln und leere Phrasen wie zum Beispiel: ,Wir betonen ausdrücklich, daß wir nicht gegen die Forderung des christlichen Sittengesetzes kämpfen, deren Ideale zu erreichen sich jeder bemühen sollte...", womit der Herausgeber, das Wissenschaftlich-humanitäre Komitee, eine verbindliche Verbeutung gegen die im Reichtstag maßgebende Zentrumspartei und das übrige Muckertum macht“ (83). Der hier in der „Neuen Zeit“ erhobene Vorwurf trifft gewissermaßen die andere Seite des durchgängig von bestimmten Brüchen gekennzeichnete Verhältnisses zwischen Arbeiterbewegung und Homosexuellenbewegung. Während einerseits in der organisierten Arbeiterklasse die bereits oben charakterisierten Fehleinschätzungen und halbabgebauten Vorurteile relativ stabil bis zur Gegenwart hielten, wird dies andererseits ergänzt durch die mehr oder weniger reaktionären Vorbehalte der organisierten Homosexuellen gegenüber der Arbeiterbewegung. Hirschfeld brachte z. B. seine politische Fortschrittlichkeit darin zum Ausdruck, daß er sich bald nach Gründung des WHK auf dem rechten Flügel der SPD engagierte und bis zu seiner Emigration Anfang der 30er Jahre dieser Partei die Treue hielt. Kurt Hiller, ein weiterer Repräsentant der Homosexuellenbewegung und militantantimarxistischer Literat, zeichnete 1921 in seinem Buch „\$175 - die Schmach des Jahrhunderts" ein drastisches Bild vom politischen Zustand der Homosexuellen:

„Ein Führer im Befreiungskampf, ein Praktiker, der es wissen muß, versicherte mir kürzlich, $75 \%$ der am Kampf Interessierten in Deutschland rechneten sich den Rechtsparteien, der monarchistischen Restauration und der Revanche, zu. Nur etwa ein Viertel der Beteiligten sei schwarzrotgold oder rot" (84).

In dem gleichen Buch tritt Hiller dafür ein, daß die Befreiungsbewegung der Homosexuellen ,parteipolitisch neutral“" zu sein habe:

„Der Royalist muß ihr als Helfer ebenso willkommen sein wie der sozialistische Republikaner, der strenggläubige Katholik wie der anarchistische Freigeist, der Kommunist wie der bürgerliche Demokrat" $(85)$.

Hatte sich neben dem auf demokratische Reformen orientierten. WHK schon früh eine kleinbürgerlich-anarchistische Strömung entwickelt (86), deren Einfluß jedoch

83 Die Neue Zeit, Jg. 20, 1901-02, Bd. 2, Nr. 29, S. 89

84 Kurt Hiller, $\S 175$ - die Schmach des Jahrhunderts, Hannover 1922, S. 78

85 a. a. O., S. 79

86 Der Literat Adolf Brand brachte 1896 die erste Homosexuellenzeitschrift heraus (,DDer Eigene"), die zunächst eine stirnerianische Position vertrat, später einen ,freiheitlichen Sozialismus“, der ,nicht dogmatisch sondern erdgeboren“ ist, ,nicht das Privateigentum verbietet" und den Klassenkampf ,,verwirft" (vgl. Unser Bekenntnis zur Republik, in: 
verschwindend gering blieb, so kam die extreme Rechte erst in den 20er Jahren organisatorisch zum Zuge. Es kam zur Gründung ,nationaler Invertiertenvereine" (87), die aber im Vergleich zum WHK ähnlich einflußlos blieben wie der Anarchismus.

Es gibt einen Anhaltspunkt dafür, daß im WHK, gemäß seiner ,,parteipolitischen Neutralität", auch homosexuelle Nazis organisiert waren: Es heißt in den „Mitteilungen“" des WHK, Nr. 8 vom Juli/August 1927 anläßlich einer antihomosexuellen und Hirschfelds jüdische Herkunft anprangernden Rede eines NSDAP-Abgeordneten im Reichstag:

„Wir fühlen uns weiter verpflichtet, unsere zahlreichen Mitglieder in der Nationalsozialistischen Deutschen Arbeiterpartei und in der Deutschvölkischen Freiheitspartei dringend aufzufordern, ihren Abgeordneten energisch zur Ordnung zu rufen“" (88)

Es liegt nun nahe, diese Tatsachen im Sinne einer geheimen Verwandschaft zwischen Homosexualität und Faschismus zu deuten. Wenn etwa auf das schlecht verdrängte homosexuelle Potential der Faschisten als ein Grund für ihren gesteigerten Homosexuellenhaß hingewiesen wird, so ist dieser Gedanke wohl nicht von der Hand zu weisen, obgleich dies auch als Merkmal jeder Zwangsheterosexualität und nicht nur der der Faschisten interpretiert werden kann. Darüber hinausgehende Zusammenhänge zwischen Faschismus und Homosexualität zu konstruieren, ist jedoch ähnlich abgeschmackt, wie wenn man z. B. Frauen eine besondere Affinität zum Rechtsradikalismus unterstellen wollte, weil Frauen nachweisbar unter der Wählerschaft der NSDAP und der CDU überrepräsentiert waren. Solchen Konstruktionen liegt letztlich die Unfähigkeit zugrunde, die Stufen der Bewußtseinsbildung von zusätzlich unterdrückten Gruppen als historische Übergangsformen zu begreifen; empirische Sachverhalte werden als ewige Gesetzmäßigkeiten dargestellt (89).

Der Eigene, Jg. 1926, Nr. 4). Brands Organisation, die „Gemeinschaft der Eigenen“ kämpfte gegen das WHK und den zunehmenden Einfluß der Frau in der Gesellschaft und sympathisierte später offen mit dem Faschismus.

87 Vgl. Jahrbuch für sexuelle Zwischenstufen, Jg. 20, 1920, S. 135

88 Zit. nach Richard Linsert, Kabale und Liebe, über Politik und Geschlechtsleben, Berlin 1931, S. 156

89 Berühmtes Beispiel für einen solchen Standpunkt ist die vielzitierte Äußerung des sowjetischen Schriftstellers M. Gorki, der in seinem Aufsatz „Proletarischer Humanismus" am 23. 5. 1934 in Pravda und Izvestija schrieb: ,[ . . ] hunderte Tatsachen sprechen von dem zerstörenden, zersetzenden Einfluß des Faschismus auf die Jugend Europas. Es widerstrebt einem, TAtsachen anzuführen; [ . . ] Ich weise jedoch darauf hin, daß Homosexualität [ ...] im ,Kultur'-Land der großen Philosophen, Gelehrten und Komponisten frei und ungestraft ist. Es ist sogar das sarkastische Sprichwort entstanden: ,Rottet die Homosexuellen aus - und der Faschismus verschwindet'." 


\section{Zum gegenwärtigen Verhältnis zwischen Sozialisten und Homosexuellen}

Bis 1969 wurde in Westberlin und in der BRD noch der $\S 175$ in der aus der Nazizeit stammenden Form angewandt. Die schließliche Lockerung der Strafbestimmung durch die SPD-CDU-Regierung 1969 war nicht von einer homosexuellen Emanzipationsbewegung erkämpft worden. Eine solche war schon aufgrund des bis dahin herrschenden antihomosexuellen Terrors, dessen Schärfe den der Weimarer Republik weit übertraf, in ihrer Entwicklung stark gehemmt. Die verschiedenen publizistischen und organisatorischen Initiativen erreichten bei weitem nicht das Niveau der Zeit vor dem Hitlerfaschismus.

Neben einer Spätwirkung der Anstrengungen des WHK in der Zeit bis 1933 sind anscheinend zwei weitere Momente für die 1969er Reform des $\S 175$ mågebend gewesen: die schon erwähnte Lockerung der Strafbestimmung in anderen Ländern - vor allem 1968 in der DDR - waren hier ebenso von Einfluß wie die beständige Intervention einzelner Individuen, z. B. die Aktivitäten des Sexualwissenschaftlers H. Giese und des Juristen J. Baumann u. a. (90).

Die seit 1969 bestehende Straffreiheit für homosexuellen Verkehr unter Erwachsenen (91) war die Voraussetzung für die allmählich sich entfaltende neuere Homosexuellenbewegung.

Obwohl in der Studentenrevolte der 60er Jahre in besonderem Maße die Frage nach der Funktion der Sexualunterdrückung im Kapitalismus gestellt wurde, geriet das Problem der Homosexuellenunterdrückung allenfalls verzerrt ins Blickfeld der Beteiligten. Das hatte nicht zuletzt seinen Grund in der mehr oder weniger subtil homosexualitätsfeindlichen Einstellung der wichtigsten Ideologielieferanten jener Phase: W. Reich und H. Marcuse. Es war sicher ein Verdienst von W. Reich, in seinem Buch „Die sexuelle Revolution“ schon früh den in der Sowjetunion der 30er Jahre beginnenden Terror gegen Homosexuelle, der mit einer allgemein zunehmenden Sexualfeindlichkeit einherging, angeprangert zu haben. Dies zwang Reich jedoch überhaupt nicht, seine eigene, aus seiner eigentümlichen Freud-Rezeption resultierenden antihomosexuelllen Vorurteile zu revidieren.

Diese Vorurteile sind klassisch in dem Abschnitt ,Zur Frage der Homosexualität“ in seiner Broschüre „Der sexuelle Kampf der Jugend“ zusammengefaßt. Homosexualität ist für Reich eine „Krankheit", vor der vor allem natürlich ,die Jugendlichen“" geschützt werden müssen:

90 Vgl. z. B. Eingabe an die gesetzgebenden Organe des Bundes in Bonn betreffend $\S 175$, 175 a StGB. Institut für Sexualforschung, Frankfurt a. M. 1950

91 Seit dem 1.1.1974 ist das antihomosexuelle Sondergesetz noch etwas weiter eingeschränkt: Während z. B. ein Mann, der eine Frau ,,zum Beischlaf verführt“ ( $\$ 182$ StGB), die das 16. Lebensjahr noch nicht vollendet hat, nur ,, auf Antrag der Eltern oder des Vormundes ... mit Freiheitsstrafe bis zu einem Jahr bestraft" wird, wird ein Mann, der ,sexuelle Handlungen an einem Mann unter 18 Jahren vornimmt ... mit Freiheitsstrafe bis zu fünf Jahren ... bestraft" ( $\$ 175 \mathrm{StGB})$. Der Kampf für die vollständige Beseitigung des $\S 175$ bleibt eines der zentralen Ziele der Homosexuellenbewegung. 
„Vor allem müssen die Jugendlichen vor der endgültigen Wendung zur Homosexualität bewahrt werden, nicht aus moralischen, sondern aus rein sexualökonomischen Gründen; denn es läßt sich feststellen, daß die durchschnittliche sexuelle Befriedigung beim gesunden, andersgeschlechtlich gerichteten noch immer viel intensiver ist als die Befriedigung bei [physisch] gesunden Homosexuellen."

In seiner "Massenpsychologie des Faschismus" kommt dementsprechend Homosexualität allein vor zum Zweck der Denunziation derer, die unter dem Einfluß faschistischer oder religiöser Ideologien stehen. Sie seien von einer ,passiven und masochistischen Homosexualität" gekennzeichnet (vgl. besonders S. $218 \mathrm{f}$. und 262 f.).

H. Marcuse läßt sich nicht so offen wie W. Reich auf das Thema ein. Tabuisierung ist seine vorherrschende Haltung zur Homosexualität. Er handelt sie ab unter dem Thema „Perversionen“ in seinem Erfolgswerk „Triebstruktur und Gesellschaft", S. 54 f.: Nach seiner Auffassung ,verteidigen die Perversionen die Sexualität als Zweck an sich; sie stellen sich damit außerhalb des Herrschaftsgebietes des Leistungsprinzips und bedrohen es in seinen Grundfesten." Für Marcuse ist diese Bedrohung jedoch so fundamental, daß er das Aussterben der Menschheit als Mögliclikeit vorausahnt:

„Außerdem würden Konzessionen gegenüber der Ausübung ₹on Perversionen nicht nur die ordnungsgemäße Fortpflanzung der Arbeitskräfte, sondern vielleicht sogar der Menschheit selbst in Gefahr bringen."

Wenn man bedenkt, daß solche Ansichten in weiten Kreisen der Linken bereitwilligg akzeptiert wurden und zum Teil noch werden, dann kann das vorhandene und von mehr oder weniger gut rationalisierten Ängsten genährte antihomosexuelle Vorurteil nicht weiter erstaunen.

Die einzige uns bekannte Ausnahme bildeten die Antworten einiger Sprecher der sogenannten $\mathrm{APO}$ wie Ulrike Meinhof, Christoph Ehmann, Nils Kaḍitzke und einiger Hochschullehrer wie T.W. Adorno, Wolfgang Abendroth und H. Gollwitzer auf eine entsprechende Umfrage, die in dem Buch ,Weder Krankheit noch Verbrechen. Plädoyer für eine Minderheit" (Hamburg 1969) veröffentlicht wurden und die relativ vorurteilsfrei ausfielen. Es ist jedoch nichtsdestoweniger zunächst erstaunlich, daß gewissermaßen als Spätprodukt dieser Studentenrevolte seit 1971 in verschiedenen westdeutschen Universitätsstädten und in Westberlin homosexuelle Aktionsgruppen entstanden, die die Homosexuellenemanzipation als Moment einer sozialistischen Umwälzung begreifen.

Das Entstehen der neueren Homosexuellen-Organisationen muß im Zusammenhang mit entsprechenden Ereignissen vor allem in USA und Großbritannien gesehen werden. Die von dort ausgehenden, seit Beginn der 60er Jahre - vor allem vermittelt über bestimmte Zweige der Unterhaltungsindustrie - sich vollziehenden Veränderungen, die oft mit kulturpessimistischem Akzerıt als ,Nivellierung der Geschlechterspannung" beschrieben wurden, bildeten wichtige Randbedingungen für

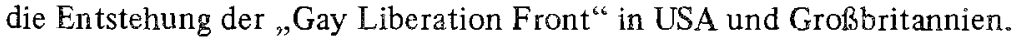

Ein weiterer wichtiger Faktor in diesem Rahmen war eine in den USA ent- 
standene Richtung innerhalb der bürgerlichen Kunstavantgarde der 60er Jahre, die vom etablierten Kunstbetrieb bisher tabuisierte Bereiche, u. a. die homosexuelle Subkultur für die Kunstproduktion entdeckte bzw. ein Teil von ihr war. In den Filmen des „New American Cinema“, vor allem in den Filmen Andy Warhols und Shirley Clarkes („Portrait of Jason") stellten sich zum ersten Mal homosexuelle Männer und Frauen selbstbewußt einer bbreiten Öffentlichkeit dar; der ästhetische Reiz von Transvestiten wurde bewußt eingesetzt. Hiermit war im Bezug auf ein Massenmedium das Tabu der Selbstdarstellung Homosexueller durchbrochen. Warhols Filme wurden zwar anfänglich nur von einem kleinen Kreis Interessierter gesehen, sie waren aber gerade für das neu entstehende Selbstverständnis vieler Homosexueller richtungsweisend. In Westdeutschland waren diese Filme sogar erfolgreicher als in USA, was sehr bald zu ihrer profitmäßigen Vereinnahmung durch die größte westdeutsche Filmverleih-Firma führte. Nachdem hiermit zum ersten $\mathrm{Mal}$ in größerem Ausmaß Homosexuelle sich selbst in einem Massenmedium darstellten, und damit zum Abbau des Homosexualitätstabus beitrugen, waren Auseinandersetzungen mit der Polizei nach Räumung eines Homosexuellen-Lokals in New York im Juni 1969 („Stonewall Riots") der unmittelbare Anlaß zur Gründung von Homosexuellenorganisationen in den USA in bisher noch nicht dagewesenem Ausmaß. Die amerikanische „Gay Liberation"-Bewegung - inzwischen auch in vielen kleinen Städten aktiv - hat überwiegend Selbsthilfefunktionen, leistet aber auch Aufklärung in der Öffentlichkeit im Rahmen der Bürgerrechtsbewegungen. Eines ihrer Hauptziele, die Beseitigung der antihomosexuellen Strafgesetze, wurde aber bisher nur in den wenigsten Bundesstaaten verwirklicht.

Die amerikanische und englische "Gay Liberation Front" war den meisten Gründungsmitgliedern westdeutscher Homosexuellengruppen zumindest dem Namen nach bekannt und wurde vor allem mittels ihrer Publikationen zur Kenntnis genommen. Sie hat so noch immer, trotz gewisser krisenhafter Veränderungen (92), einen Einfluß auf die deutschen Gruppen.

Eine wichtige Funktion für die Gründung der meisten deutschen Gruppen hatte der von dem homosexuellen Filmregisseur Rosa von Praunheim und dem ebenfalls homosexuellen Soziologen Martin Dannecker stammende Agitationsfilm "Nicht der Homosexuelle ist pervers, sondern die Situation, in der er lebt" (93). Obwohl in diesem Film selbst die politischen Konsequenzen nur äußerst vage angedeutet werden, entwickelten viele der Gruppen doch richtige Ansätze zur Einschätzung ihrer Lage. So heißt es z. B. in der im November 1971 verabschiedeten Grundsatzerklärung der Homosexuellen Aktion Westberlin:

92 Vgl. hierzu für Großbritannien: David Fernbach, Aufstieg und Fall der GLF (The rise and fall of GLF, deutsch), in: HAW-Info, Nr. 14 1974, S. 28 ff.

93 Magnus Hirschfeld versuchte schon 1919, allerdings mit viel geringerem Erfolg, den Film für den homosexuellen Emanzipationskampf einzusetzen. Sein Film ,Anders als die Andern", der zum Kampf gegen den $\S 175$ aufrief, wurde bald von der Polizei verboten. Rosa von Praunheim und Dannecker knüpften jedoch nicht bewußt an điesen Vorläufer an, sondern empfingen wohl eher von den Filmen des erwähnten New American Cinema ihre Anregungen. 
„Der Rahmen, d. h. die Möglichkeiten der Emanzipation der Homosexuellen ist vorgezeichnet durch die kapitalistisch organisierte Gesellschaft. Selbstbestimmung für alle ökonomisch wie sexuell Unterdrückten ist nur in geringen Ansätzen zu verwirklichen, da unter der Herrschaft des Kapitalinteresses die Organisation gesellschaftlichen Lebens unter dem Primat der Profitmaximierung erfolgt. Die Produzenten des gesellschaftlichen Reichtums, der im Kapitalismus privat angeeignet wird, werden sowohl von der Organisation der Produktion als auch von der Verfügung über den gesellschaftlichen Reichtum ferngehalten [...] Der Kampf der Homosexuellen um Selbstbewußtsein und Identität setzt voraus, daß sie ihre Existenz als Homosexuelle mit ihrer ökonomisch-politischen Existenz vermitteln."

Solche Einsichten, die durchaus auch praktische Konsequenzen zeitigten (Unterstützung des antiimperialistischen Kampfs, Teilnahme an Demonstrationen zum 1. Mai, Chile-Solidarität) geraten jedoch ständig in Gefahr, wieder vergessen zu werden. Der Rückfall in ein unpolitisches Selbstverständnis, das Aufgreifen bürge rlicher Modeideologien sind ständig gegenwärtige Alternativen zu einer konsequent sozialistischen Orientierung. Dies gilt vor allem für die Zeit der anfänglichen Konsolidierung, die durchaus noch nicht abgeschlossen ist. Ein Beispiel für solche mögliche Fehlentwicklung bietet augenblicklich die Frauengruppe der HAW: mit der Doktrin „Feminismus ist unsere Theorie, lesbisch sein ist unsere Praxis"versuch te die Frauengruppe nicht nur ihre Absonderung von den homosexuellen Männern, sondern auch ihre so gut wie vollständige Enthaltsamkeit auf politischem Gebiet zu rechtfertigen; politische Praxis beschränkt sich für sie seit einiger Zeit fast nur noch auf einen vermeintlichen Kampf gegen Männerherrschaft.

Einer der wichtigsten Faktoren für die ideologisch-politische Entwicklung der homosexuellen Aktionsgruppen ist die Einstellung der sozialistischen Organisationen zur Homosexuellenunterdrückung. Auf die eigentümlichen Fehleinschätzungen, die in der Vergangenheit vorherrschten, sind wir hier eingegangen. Daß sich gegenwärtig daran kaum etwas geändert hat, muß leider konstatiert werden. Beispielsweise war die Verlautbarung der maoistisch orientierten Studentengruppe KBW gegenüber der Homosexuellen Aktion Bremen,wonach organisierter Kampf Homosexueller gegen ihre Unterdrückung ein „Unsinn“ sei und eine ,bürgerliche Einrichtung“, von der sich gute Kommunisten fernzuhalten hätten (94), für die Bremer Gruppe ähnlich demoralisiczend wie die bereits zitierte Stellungnahme der SEW in ihrer „Wahrheit“ für die Hon osexuelle Aktion Westberlin.

$\mathrm{Da} ß$ solche Erfahrungen im Extremfall bereits bis zur Wiederbelebung abgeschmacktester Eliteideologien geführt haben, zeigt das oben schon erwähnte Beispiel Hocquenghems, eines äußerst populären Ideologen der Homosexuellenbewegung in Frankreich: Im gleichen Atemzug mit der Glorifizierung der Homosexuali-

94 Vgl. die ,Dokumentation zur Auseinandersetzung über die besondere Unterdrückung der homosexuellen Arbeiter und Werktätigen", hrsg. von der Homosexuellen Aktion Bremen im März 1974. Sie enthält den vollständigen Text der von W. Maier im Auftrag des KBW abgegebenen Stellungnahme zur Homosexuellenunterdrückung. Für W. Maier ist eine Homosexuellengruppe ,eine direkt konterrevolutionäre Organisation, weil sie gegen die Scheidung der Gesellschaft nach Klassen, die Zusammenführung verschiedener Klassenbestandteile nach dem Charakter der sexuellen Bedürfnisse setzt". Nach dieser abstrusen Logik Maiers wäre z. B. auch ein kommunistischer Jugendverband, ein kommunistischer Frauenbund, Schriftstellerverband usw, „,konterrevolu tionär". 
tät, der er eine ,ungeheure Überlegenheit" nachsagt, konstatiert er verbittert, die von der ,extremen Linken“ bis hin zur FKP zum Ausdruck gebrachten antihomosexuellen Vorurteile, die noch immer so weit gehen, in der Homosexualität eine besonders raffinierte Waffe des Klassenfeindes zu vermuten (95).

Es sind zur Zeit keine Anzeichen dafür in Sicht, daß sozialistische Organisationen ihre Fehleinschätzungen bzw. das Tabu zu dieser Frage zu revidieren bereit sind. Dies wird langfristig unweigerlich Auswirkungen auf die homosexuelle Emanzipationsbewegung haben. Der Rückfall in Anschauungen, die bis zu kleinbürgerlich-anarchistischen Ideologievarianten und zur weitgehenden Entpolitisierung reichen, würden beschleunigt fortschreiten. Die Stagnation des Verhältnisses zwischen Homosexuellenbewegung und Arbeiterbewegung wäre das Resultat.

Die einzige sinnvolle Perspektive, die allmähliche Integration der Homosexuellenbewegung in die Arbeiterbewegung als Konsequenz eines wirklich vorurteilsfreien Verhältnisses zur homosexuellen Minderheit wäre wieder einmal verloren.

\section{Praktische Konsequenzen}

Wenn es zutrifft, wie hier zu zeigen versucht wurde, daß die Sonderunterdrückung der Homosexualität kein Wesensmerkmal der kapitalistischen Gesellschaft ist; daß dieses aus vorkapitalistischen Epochen überkommene Phänomen in den Anfängen des Kapitalismus durchgängig und mit weiterer Entwicklung mehr und mehr nur noch sporadisch und reduziert anzutreffen ist; daß also die Diskriminierung homosexuellen Verhal tens - bedingt durch dem Kapitalismus immanente Faktoren - in einem Angleichungsprozeß dieser besonderen an die allgemeine Unterdrückung der Ausgebeuteten, die nach anderen Kriterien als dem der sexuellen Orientierung differenziert ist, aufgelöst wird, dann lassen sich daraus einige allgemeine Bestimmungen über Reichweite und Verlaufsform des Emanzipationskampfs der homosexuellen Männer und Frauen ableiten, deren Nichtbeachtung zu durchaus vermeidbaren Umwegen und Rückschlägen führen muß:

1. Emanzipationsforderungen der Homosexuellen können den Rahmen der bürgerlichen Gesellschaft nicht infrage stellen. Auch die scheinbar radikalste Forderung nach einer Auflösung der tradierten und starr entgegengesetzten Geschlechterrollen, die die Tabuisierung gleichgeschlechtlicher Sexualität beinhalten, sind in der bürgerlichen Gesellschaft prinzipiell verwirklichbar und allenfalls insofern utopistisch, als derartige Veränderungen des Sexualverhaltens irn Massenmaßstab nicht über Propaganda oder beispielhafte Aktionen bewirkt werden. Es sind dies bestenfalls Momente in einem vom Kapitalismus selbst produzierten Prozeß der Auflösung altertümlicher Verhaltensnormen, die an ein mehr oder minder dysfunktional werdendes System patriarchalisch geprägter Geschlechtsrollen gebunden sind und in ein System der Sexualunterdrückung transformiert werden, das dem derzeitigen Kapitalismus besser 
entspricht. Die Analyse dieser sich abzeichnenden neuen Formen der Sexualunterdrückung ist noch kaum in Angriff genommen worden.

2. Der Emanzipationskampf der Homosexuellen muß in den Bereichen, die sich auf die Sexualität beschränken (Strafrecht; Diskriminierung am Arbeitsplatz, in der Öffentlichkeit usw.) in einem breiten Bündnis geführt werden, das auch die Homosexuellen mit einbezieht, die die Notwendigkeit einer sozialistischen Umwälzung noch nicht erkennen. Konzepte, die von einer die Gesellschaftsordnung sprengenden Kraft des Emanzipationskampfs der Homosexuellen als Homosexuelle ausgehen, verkennen die Grenzen und Möglichkeiten des Kapitalismus und sind in doppelter Weise ,sektiererisch“. Zum einen führen sie spätestens dann zur Isolation von der breiten Masse der Homosexuellen, wenn diese nicht die Homosexualität, sondern die Ausbeutung ihrer Arbeitskraft als den entscheidenden Punkt ihrer Unterdrückung erkennen; zum anderen verhindern derartige Konzepte ein Aufgehen der Homosexuellenbewegung in die Arbeiterbewegung, es sei denn um den abwegigen Preis, daß die Arbeiterbewegung die vermeintlich grundlegende Bedeutung der derzeitigen Formen der Sexualunterdrückung für den Klassenkampf anerkennen würde.

3. Auch wenn man sich nicht der Illusion hingibt, daß der Kampf gegen Homosexuellenunterdrückung zentrale Momente einer allgemeinen sexuellen Befreiung im Sozialismus-Kommunismus antizipiert, ist dieser Kampf immer noch dann borniert, wenn in der Taktik die wesentliche, $d$. h. die für den Kapitalismus existentiell notwendige Unterdrückung vernachlässigt wird: die Unterdrückung und Ausbeutung der Homosexuellen als Lohnarbeiter. Ist eine bürgerliche Homosexuellenbewegung durch diese Borniertheit gekennzeichnet, so ist der entscheidende Punkt für sozialistische Homosexuelle die Einsicht in den Primat des Klassenkampfes: die Einsicht, daß die Aufhebung der Homosexuellenunterdrückung kein Ersatz ist für die soziale Emanzipation der Arbeiterklasse, daß also die Befreiung der Homosexuellen wie der Heterosexuellen nur im Sozialismus verwirklicht werden kann.

4. Da die Illusionen der Homosexuellen und die Borniertheit ihres Kampfes doppelt determiniert sind - durch den Fortschritt im Abbau der zusä tzlichen Homosexuellenunterdrückung durch objektive Veränderungen im Kapitalismus und durch den Fortschritt im Abbau der antihomosexuellen Vorurteile bei Sozialisten -, besteht eine zentrale Aufgabe homosexueller Sozialisten darin, ihre Selbstverleugnung zu überwinden und die antihomosexuellen Vorurteile, die Ängstlichkeiten und Tabus der heterosexuellen Genossen offensiv zu kritisieren. Dies wird ihnen jedoch nur in dem Maße gelingen, wie sie Rückhalt in einer breiten Homosexuellenbewegung finden.

5. Solange unter Sozialisten nicht der radikale Bruch mit den eigenen antihomosexuellen Vorurteilen vollzogen wird, wobei es nebensächlich ist, ob homosexuelles Verhalten als Krankheit, Verbrechen oder „Dekadenz“ aufgefaßt wird; solange Sozialisten - oft sogar bewußt - dazu beitragen, die homosexuellen Männer und Frauen ins Getto abzudrängen und sich nicht wenige Homosexuelle in den Reihen der Sozialisten zur Selbstverleugnung und zum 
menschenunwürdigen Doppelleben gezwungen sehen, weil ihr „Privatproblem" bestenfalls Mitleid erregt; solange ist die Chance der konkreten Erkenntnis einer sozialistischen Perspektive für Homosexuelle viel geringer als für Heterosexuelle und die Gefahr des Rückzugs in Resignation oder in scheinradikale Trost- und Eliteideologien, die die homosexuelle Emanzipationsbewegung auf die Dauer paralysieren oder bestenfalls zu Bürgerrechtsvereinen verkommen lassen, nicht abgewendet. Die aktive Solidarität der Sozialisten mit den gegen ihre Unterdrückung kämpfenden homosexuellen Männern und Frauen muß zur Selbstverständlichkeit werden. 


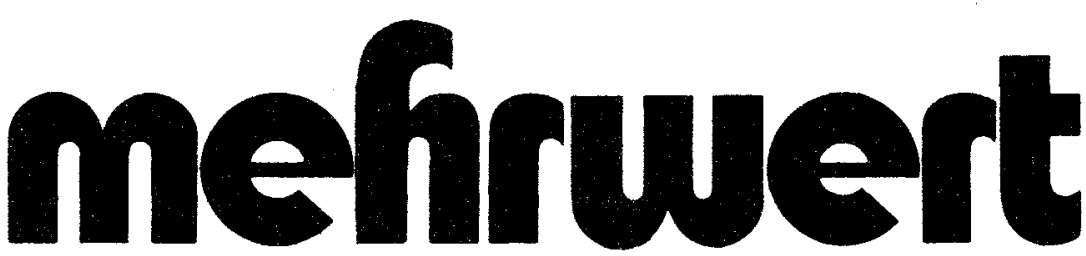

BEITRÄGE ZUR KRITIK DER POLITISCHEN ÖKONOMIE mehrwert 1

192 Seiten DM 8,00

Gesamtwirtschaftliche Grundbegriffe und -beziehungen. Wert- und Preislehre; Studienmaterialien, I. Teil

mehrwert 2

176 Seiten DM 7,00

Zur Kritik der bürgerlichen Wirtschaftstheorie

Zur Interpretation der Marxschen Reproduktionsschemata (mit einem An= hang von Jörg Glombowski)

Wert und Preis in der marxistischen und neokeynesianischen Akkumulationstheorie

mehrwert 3

278 Seiten DM 10,00

Einzelwirtschaftliche Grundbegriffe und -beziehungen. Zur Kritik der Betriebswirtschaftslehre. Teil I: Die Produktion

mehrwert 4

126 Seiten DM 6,50

Die Theorie des staatsmonopolistischen Kapitalismus - Kritik der zentralen Aussagen

Sozialistische Warenproduktion - Warenproduktion im Sozialismus. Ein Vergleich dreier Bücher.

mehrwert 5

190 Seiten DM 8,00

Zur Struktur des Krisenproblems bei Karl Marx

mehrwert 6

144 Seiten DM 8,00

Joan Robinsons Thesen zum Verhältnis von Keynesscher und Marxscher Theorie

Das Gesetz des tendenziellen Falls der Profitrate - Marx's Begründung und ihre Implikationen

Arbeiterstandpunkt und politische Ökonomie

Die systemtheoretische Entproblematisierung der marxistischen Gesellschaftstheorie

\section{mehrwert 7}

176 Seiten DM 9,00

Weltgeld und Wertgesetz. Zur Ersetzbarkeit der Geldware durch Zeichengeld. mehrwert 8

212 Seiten DM 12,50

Gesamtwirtschaftliche Grundbegriffe und -beziehungen. Akkumulation und Beschäftigung; Studienmaterialien, II. Teil

Die Schriftenreihe Mehrwert erscheint in unregelmäßigen Abständen. Der Preis variiert je nach Umfang und Auflage.

\section{Erhältlich in den Buchläden oder portofrei per Post durch POLITLADEN ERLANGEN 852 Erlangen Postfach 2849}




\section{im focus: im focus im focus:}

\section{Portugal}

Materialien und Dokumente

herausgegeben und übersetzt von:

\section{Günter Schröder}

Im Focus-Verlag ist eine umfassende Dokumente- und Materialiensommlung zu den Ereignissen in Portugol seit dem 11. Marz 1974 bis hin zum sozialdemokratischen Putsch vom 25. November 1975 erschienen. Die Dokumente sind eingeleitet, kommentiert und aus dem Portugiesischen ubersetzt von GUNTER SCHROODER und beinhalten im wesentlichen Stellungnahmen und Informationen zur Politik der Organisationen zwischen Revisionisten und Marxisten-Leninisten, also MES, LUAR, FSP und PRP-BR, sowie Dokumente des MFA und der BasisBAND 1 BAND 2 organisationen. enthalten Dok. ous dem Zeitraum Màrz 175 bis 9.Juli 1975. Sie beziehen sich auf die Bereiche: Institutionalisierung des MFA in Politik und Streitkraften, Aufbau von Poder Popular, Verbindung MFA - Poder Popular in der Allianz Volk MFA. Dazu Dok. zur Politik des PS, zum politischen Aktionsplan des MFA und zu den Auseinandersetzungen $z w$ ischen MRPP und COPCON. Zum besseren Verstandnis der Ausgangslage des MFA wurde das MFA-Programm vom 25.4.74 den übrigen Dokumenten vorangestellt.

Band 1: 168 Seiten, DM 7.-

Band 2: 200 Seiten, DM 8.50

BAND 4

bezieht sich auf den Zeitraum 5.9. - 25.11. d.h. vom Sieg der sozioldemokratischen Militärs im MFA zum sozioldemokratischen Putsch vom 25.11.1975.Die Dok. beschreiben die Auseinandersetzungen in den Streitkraften, die Schwierigkeiten beim Aufbau von Poder Popular und der Schaffung uberortlicher Koordinationsgremien und Stellungnahmen von linkssozialistischen Organisationen zu kon- AUSLIEFERUNG: kreten Ereignissen und zur politischen Lage. ca. 200 Seiten (einzeilig), ca. DM 9.-
FOCUS - Verlag GmbH 6300 Giessen

Dammstr.11/Postf. 2328

Tel. $0641 / 34760$
BAND 3

bezieht sich auf den Zeitroum $z$ wischen der Verabschiedung des Leitfadens der Allianz Volk-MFA (9.7.) und der Versammlung des MFA in Toncos. (5.9.), ouf der die militärische Sozialdemokratie in Gestalt der "Gruppe der Neun" einen entscheidenden Durchbruch gegenüber den sozialistischen Kraften erzielte.

Es werden die wichtigsten Ereignisse auf politischmilitärischer Ebene in diesem Zeitraum beschrieben: Reaktinàre Gegenoffensive unter Fuhrung des PS, Abwehrversuche der sozialistischen Kräfte, Zerfall des Mehrheitsbundnisses im MFA und Durchbruch der Sozialdemokrotie mit $\mathrm{Hilfe}$ der rechten Krafte in Heer und Luftwaffe.

197 Seiten (einzeilig) DM 9.-

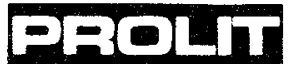

Buchversand 63 obesen Dommsti.
Postiacs

\section{im focus: im focus: im focus:}




\section{olle und woller}

Richard Müller

GESCHICHTE DER DEUTSCHEN REVOLUTION ( 3 BÄNDE)

Die klassische Chronik der Novemberrevolution und ihrer Vorgeschichte hat kein professioneller Historiker, sondern einer geschrieben, den sich die Arbeiter selber an ihre Spitze gestellt haben. Der 'Rätemüller' schildert die Streikbewegung im Weltkrieg, die Errichtung der Räterepublik und ihre Niederwerfung im sozialdemokratischen Belagerungszustand als Problem, wie eine revolutionäre Taktik die Massen ergreifen kann. Stellt die sozialistische Literatur diesen Zeitabschnitt gewöhnlich als Geschichte der Führer und Verräter dar, so lehnt sich Müller sichtlich an Marx' eigene Geschichtsschreibung an, die die Klassenbewegung nicht aus der Führung, sondern die Führungen aus der Klassenbewegung erklärt, die in ihrer verwickelten Dynamik analysiert sein will.

Band 1: Vom Kaiserreich zur Republik, 220 Seiten, DM 8,80

Band 2: Die Novemberrevolution, 296 Seiten, DM 12,80

Band 3: Der Bürgerkrieg in Deutschland, 260 Seiten, DM 9,80

KRITISCHE BIBLIOTHEK DER ARBEITERBEWEGUNG

Bei Bestellungen an den Verlag plus DM 1.- Porto, bei Gruppenbestellungen ab 10 Ex. 10\% Rabatt, Liefferung erst nach Eingang der entsprechenden Summe auf unserem Konto. Uber weitere Titel der Reihe EDIfION PRINKIPO und KRITISCHE BIBLIOTHEK DER ARBEITERBEWEGUNG, wie der Zeitschrift KRITIK DER POLITISCHEN ÓKONOMIE informiert unser Prospekt, der angefordert werden kann (bitte $40 \mathrm{pfg}$. Briefmarken beilegen).

Verlag Olle \&. Wolter, 1 Berlin 30, Postfach 4310, Postscheckkto. BInW 47006-104, Sparkasse Berlin West, Kto.Nr. 067001142 


\section{Peilhe \\ ROMANE \\ REPORTAGEN \\ Alilobio- \\ giraphion}

Trikonl

8Munchen 80

Josephsburgstr. 16

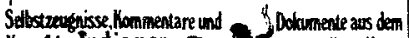

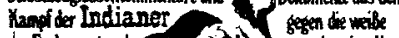
Enternas und

Die Wunden der Freiheit

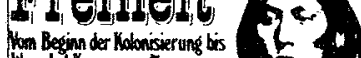

the

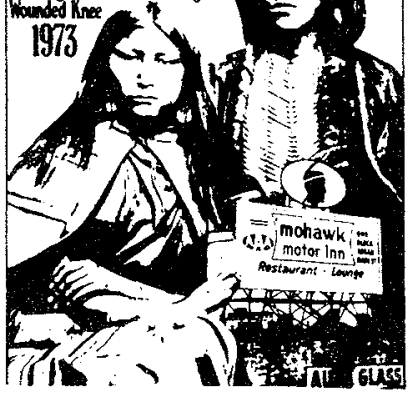

\section{Mein Folk soll l leben Reila Khaled}
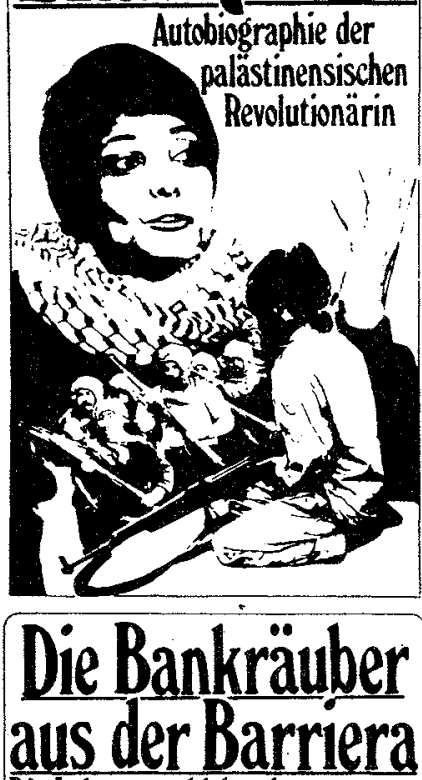

Die Lebensgeschichte des

Revolutionars Sante Notarnicola von ihm selbst aufféschrieben.

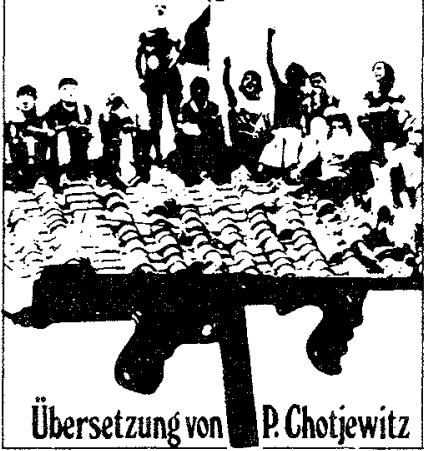

Louis Adamic

Geschichte des Klassenkampfes in den USA
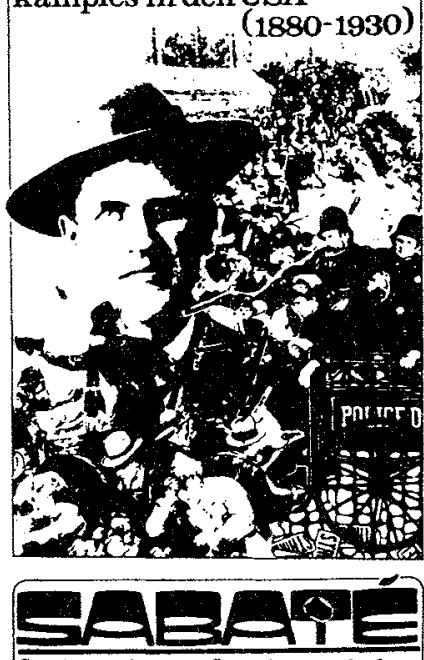

Stadtouerillia in Spanien nach dem Büngerkries 1945-1960 Aufgeschrieben von Antonjo Tellez

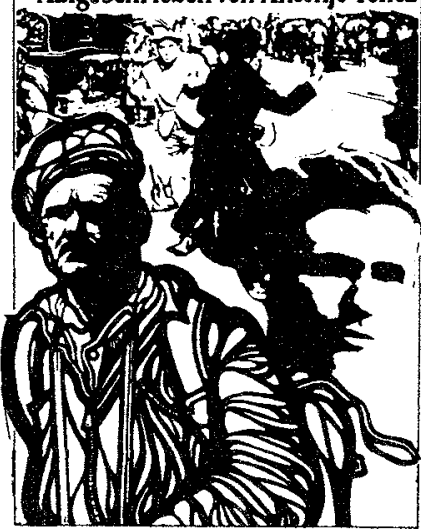

\section{Freauren.}

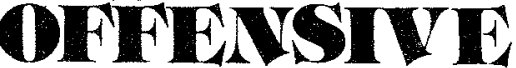

Frauen aus verschiedenen Frauengruppen geben eine eige ne Reihe heraus: FRAUENOFFENSIVE - Buchreihe Journal -- Schallplaten. Intormationen über die Frauenbewegung aus dem In- a.Ausland, feministische Andysen und subjektive Erfahrungsberichte sollen zur Diskussion gestellt und die praktischen Bewegungen unterstützt werden.
Wohnungsskampf in Frankfurt

Zehntausende lecrstehenuler Wohnun-

tel - Mieten, die niemand mehr bezahlen kann - dagegen richtet

sich der immer massenhater werdente Widerstand. Frankfurt war das Zentrum dieser Bewegung. 1)er Zusammenhang von kapiralisti cher Stadtentwicklung und der Widerstand dagegen wird hier ol ca $180 \mathrm{~S}$. 